\title{
CLINICAL TRIAL PROTOCOL
}

\author{
OLT1177-06
}

\section{A Phase 1b, Randomized, Double-Blinded, Dose Escalation, Single Center, Repeat-Dose Safety and Pharmacodynamics Study of Orally Administered Dapansutrile Capsules in Subjects with NYHA II-III Systolic Heart Failure}

\author{
Drug Development Phase: $\quad$ Phase 1b \\ Investigational product: Dapansutrile Capsules \\ Indication: $\quad$ Heart Failure \\ Sponsor: $\quad$ Olatec Therapeutics LLC \\ Protocol Version: $\quad 5.0$
}

Conduct: In accordance with the ethical principles that originate from the Declaration of Helsinki and that are consistent with International Conference on Harmonisation (ICH) guidelines on Good Clinical Practice (GCP) and regulatory requirements as applicable.

\section{CONFIDENTIAL INFORMATION}

This document is the sole property of Olatec Therapeutics LLC ("Olatec"). This document and any and all information contained herein must be considered and treated as strictly confidential. This document shall be used only for the purpose of the disclosure herein provided. Publication of any information and data is subject to the terms of the Clinical Trial Agreement for Study OLT1177-06 and will be determined in consultation with the Sponsor and Principal Investigator. 


\section{PROTOCOL APPROVAL SIGNATURE PAGE}

\section{SPONSOR: OLATEC THERAPEUTICS LLC}

I have read and understand the contents of this clinical protocol for Study OLT1177-06 (Version 5.0 dated 01 April 2019) and agree to meet all obligations as detailed in all applicable regulations and guidelines. In addition, I will inform the Principal Investigator and all other Investigators of all relevant information that becomes available during the conduct of this clinical trial.

Approved By:

\section{Damaris Skouras}

Chief Executive Officer,

Olatec Therapeutics LLC

Cynthia Barrow

Vice-President of Operations and Sponsor

Representative, Olatec Therapeutics LLC

\section{Curtis L. Scribner, MD}

Chief Medical Officer \& Medical Monitor, Olatec Therapeutics LLC

\section{Date}

Date

\section{Amy K. Poshusta, PhD}

Date 


\section{PRINCIPAL INVESTIGATOR'S AGREEMENT}

I have read and understand the contents of this clinical protocol for Study OLT1177-06 (Version 5.0 dated 01 April 2019) and will adhere to the clinical trial requirements as presented, including all statements regarding confidentiality.

By signing the protocol, I agree to keep all information provided by Olatec Therapeutics LLC ("Olatec") in strict confidence and to request the same from my personnel and the Institutional Review Board (“IRB”). Clinical trial documents provided by Olatec (protocols, Investigator's Brochure, CRFs and other materials) will be stored appropriately to ensure their confidentiality. I understand that information provided by Olatec may not be disclosed to others without direct written authorization from Olatec. In addition, I will conduct the clinical trial in accordance with current Good Clinical Practices and applicable local and FDA regulatory requirements:

Principal Investigator's Signature:

Principal Investigator's Name:

Institution:

Date: 


\section{PROTOCOL SYNOPSIS}

\section{Sponsor:}

Olatec Therapeutics LLC

Investigational Drug:

Dapansutrile Capsules
Developmental Phase: Phase $1 b$

Title of Clinical Trial: A Phase 1b, Randomized, Double-Blinded, Dose Escalation, Single Center, Repeat-Dose Safety and Pharmacodynamics Study of Orally Administered Dapansutrile Capsules in Subjects with NYHA II-III Systolic Heart Failure

Protocol Number: OLT1177-06

Indication: Safety/PK in subjects with NYHA II-III systolic heart failure

\section{Objectives:}

1. To assess the safety and tolerability of dapansutrile capsules after oral administration in subjects with chronic symptomatic, clinically stable, systolic heart failure (left ventricle ejection fraction $[\mathrm{LVEF}] \leq 40 \%$ and New York Heart Association (NYHA) functional classification II-III [NYHA II-III])

2. To assess the plasma pharmacokinetic concentrations of dapansutrile following oral administration of dapansutrile capsules

3. To assess the pharmacodynamics effect of dapansutrile following oral administration of dapansutrile capsules as measured by changes in inflammatory biomarkers

Methodology: This is a Phase $1 \mathrm{~b}$ randomized, double-blinded, single-center safety and pharmacodynamics study of sequential cohort, dose-escalating, repeat-dosing of dapansutrile or placebo (4:1 ratio) in subjects with stable systolic heart failure (HF) with $\mathrm{LVEF} \leq 40 \%$ and symptomatic for NYHA functional classification II-III who show signs of systemic inflammation (C-reactive protein [CRP] or high sensitivity plasma $\mathrm{C}$-reactive protein [hsCRP] $>2 \mathrm{mg} / \mathrm{L}$ ). A total of approximately 30 subjects will be enrolled in 3 sequential cohorts by randomized allocation (8 active and 2 placebo within each cohort). Progression to cohort 2 with dose escalation will occur following the Day 28 visit of the last subject in the first cohort.

Progression to cohort 3 with dose escalation will occur following the Day 8 visit of the last subject in the second cohort.

Subjects will be screened and evaluated twice for eligibility: 1) at the time of Screening (up to 28 days prior to randomization); and 2) at the Baseline visit, prior to randomization. At Baseline, assessments will be conducted and if the subject is eligible, the first dose of investigational product (either dapansutrile capsules or identical placebo capsules) will be administered under supervision of site personnel at the clinical site upon completion of all assessments and collection of baseline parameters. Subjects will then self-administer investigational product once, twice or four times daily, depending on cohort, for up to fourteen (14) consecutive days beginning at the Baseline visit and continuing through the planned Day 


\section{Sponsor:}

Olatec Therapeutics LLC

\section{Investigational Drug:}

Dapansutrile Capsules
Developmental Phase:

Phase $1 \mathrm{~b}$

14 visit with administration occurring under supervision of site personnel on all study treatment days involving a site visit. Subjects will return to the study clinic on Days 4, 8, 14 and 28 for follow-up visits. Additionally, it is suggested (not required) that subjects be contacted by telephone for dosing compliance confirmation approximately every 1-2 days during the treatment period. In all cases, however, subjects will be provided with 24-hour contact numbers to reach the Investigators. Subjects will also receive a follow-up telephone call on Day 42 ( \pm 3 days).

Safety and tolerability will be evaluated by monitoring the occurrence of adverse events (AEs) and changes in abbreviated physical examination findings, ECGs, vital signs and clinical safety laboratory test results (chemistry, hematology and cardiac biomarkers) and body composition measured by bio-impedance analysis. Pharmacodynamics/biomarker endpoints will include blood sampling for plasma hsCRP, IL-1 $\beta$ and interleukin-6 (IL-6), among others. Assessment of cardiorespiratory fitness will include measurement of peak oxygen consumption (peak $\left.\mathrm{VO}_{2}\right)$, and ventilatory efficiency $\left(\mathrm{VE} / \mathrm{VCO}_{2}\right.$ slope) using cardiopulmonary exercise testing (CPX). Cardiac systolic and diastolic function will be measured with transthoracic Doppler echocardiography and quality of life questionnaires (Kansas City Cardiomyopathy Questionnaire and Duke Activity Status Index). Additionally, blood samples will be collected for pharmacokinetic assessment of dapansutrile exposure and a pain questionnaire (Brief Pain Index) will be administered to capture any changes to a subject's existing aches/pains over the duration of the study.

\section{Diagnosis and Main Criteria for Inclusion:}

1) Male and female subjects 18 years old or older

2) Symptomatic stable HF (NYHA class II-III) with reduced left ventricular ejection fraction (LVEF $\leq 40 \%$, measured within 6 months of enrollment - no changes in cardiac medications or new device implantation within past 2 months)

3) Peak exercise limited by shortness of breath and/or fatigue associated with a respiratory exchange ratio (RER) $>1.00$ (reflecting maximal aerobic effort)

4) Reduced peak aerobic exercise capacity (peak $\mathrm{VO}_{2}$ ) to less than $80 \%$ of predicted value by age/gender at Baseline

5) Plasma CRP or hsCRP levels $>2 \mathrm{mg} / \mathrm{L}$ at Screening

6) Acceptable overall medical condition to be safely enrolled in and to complete the study (with specific regard to cardiovascular, renal and hepatic conditions) in the opinion of the Principal Investigator 


\begin{tabular}{|l|l|l|}
\hline Sponsor: & Investigational Drug: & Developmental Phase: \\
Olatec Therapeutics LLC & Dapansutrile Capsules & Phase $1 \mathrm{~b}$
\end{tabular}

7) Ability to provide written, informed consent prior to initiation of any study-related procedures, and ability, in the opinion of the Principal Investigator, to understand and comply with all the requirements of the study 


\section{Main Criteria for Exclusion:}

1) Women of childbearing potential, or men whose sexual partner(s) is a woman of childbearing potential who:

a. Are or intend to become pregnant (including use of fertility drugs) during the study

b. Are nursing

c. Are not using an acceptable, highly effective method of contraception until all follow-up procedures are complete. (Acceptable, highly effective forms of contraception are defined as: oral contraception, intrauterine device, systemic [injectable or patch] contraception, double barrier methods, naturally or surgically sterile, strict abstinence or partner has been sterilized. If hormonalbased birth control is being used, subject or subject's sexual partner(s) must be on a stable-dose for $\geq 3$ months prior to the Baseline visit and maintained at the same dosing level throughout the study.)

2) Abnormal blood pressure or heart rate response, angina or ECG changes (ischemia or arrhythmias) occurring during CPX

3) Presence or known history of autoimmune conditions (e.g., systemic lupus erythematosus, hypophysitis, etc.)

4) History or evidence of active tuberculosis (TB) infection at Baseline visit or one of the risk factors for tuberculosis such as but not limited or exclusive to:

a. History of any of the following: residence in a congregate setting (e.g., jail or prison, homeless shelter, or chronic care facility), substance abuse (e.g., injection or non-injection), health-care workers with unprotected exposure to subjects who are at high risk of $\mathrm{TB}$ or subjects with $\mathrm{TB}$ disease before the identification and correct airborne precautions of the subject

or

b. Close contact (i.e., share the same air space in a household or other enclosed environment for a prolonged period (days or weeks, not minutes or hours)) with a person with active pulmonary TB disease within the last 12 months.

5) Use of any prohibited concomitant medications/therapies over the periods defined in Section 5.6.3 or planned use of any prohibited concomitant medications/therapies during the Treatment Period

6) Any other concomitant medical or psychiatric condition(s), disease(s) or prior surgery(ies) that, in the opinion of the Principal Investigator, would impair the subject from safely participating in the trial and/or completing protocol requirements, including but not limited to:

a. physical inability to walk on a treadmill

b. decompensated HF (edema, NYHA IV)

c. significant ischemic heart disease, angina 
Sponsor:

Olatec Therapeutics LLC
Investigational Drug:

Dapansutrile Capsules
Developmental Phase: Phase $1 b$

d. arterial hypotension (blood pressure [BP] systolic $<90 \mathrm{mmHg}$ )

e. arterial hypertension (resting BP systolic $>160 \mathrm{mmHg}$ )

f. atrial fibrillation with rapid ventricular response

g. severe valvular disease

h. severe chronic obstructive or restrictive pulmonary disease

i. moderate-severe anemia $(\mathrm{Hgb}<10 \mathrm{~g} / \mathrm{dL})$

j. severe diabetic neuropathy or myopathy

7) Active or recent (within 2 weeks) infection prior to the Baseline visit

8) History of or known positive for HIV, Hepatitis B surface antigen or antibodies to Hepatitis C Virus

9) Known history of renal impairment and/or creatinine clearance less than $50 \mathrm{~mL} / \mathrm{min}$ calculated by Cockcroft-Gault method

10) Active malignancy or recent malignancy with chemotherapy treatment within the past 6 months

11) Enrollment in any trial and/or use of any investigational product or device within the immediate 30-day period prior to the Baseline visit

12) Previous exposure to the investigational product

\section{Dose and Mode of Administration:}

\section{Investigational Product}

Dapansutrile capsules or Placebo Capsules will be self-administered by mouth once, twice or four times depending on the Cohort, each day beginning at the Baseline visit and continuing through the planned Day 14 visit. Depending on the time of the subject's Baseline visit and Day 14 visit, all doses may not be administered on those days. The first dose of investigational product (at the Baseline visit) will be administered while in the study clinic under supervision of site personnel. Additionally, investigational product administration will occur under supervision of site personnel on all subsequent study treatment days involving a site visit.

\section{Dose Selection}

The doses selected for this trial have been qualified in preclinical studies and clinical Phase 1 pharmacokinetic and safety trials and are based on the targeted safe and effective dose for the treatment of acute gout flare (Study OLT1177-05). The dose for Cohort 1 will consist of five 100-mg dapansutrile capsules administered once a day (for a total of $500 \mathrm{mg}$ of dapansutrile drug substance per day). The dose for Cohort 2 will consist of five 100-mg dapansutrile capsules administered twice each day (BID, for a total of $1,000 \mathrm{mg}$ of dapansutrile drug substance per day). The dose for Cohort 3 will consist of five 100-mg dapansutrile capsules 
Sponsor:

Olatec Therapeutics LLC
Investigational Drug:

Dapansutrile Capsules
Developmental Phase:

Phase $1 b$

administered four-times each day (QID, for a total of 2,000 $\mathrm{mg}$ of dapansutrile drug substance per day). A clean human safety profile was previously demonstrated in the Phase 1 single and multiple dose escalation study of dapansutrile capsules (up to $1 \mathrm{~g} /$ day for 8 days) in normal healthy volunteers. Based on preclinical toxicology testing, dapansutrile has a clean safety profile to a human-equivalent dose of $10 \mathrm{~g} /$ day for up to 14 days.

\section{Concomitant Medication Use}

Concomitant medication use will be recorded and reviewed at each study visit. Subjects should remain on stable doses of allowed concomitant medications. Prohibited concomitant medications include oral corticosteroids (within two weeks prior to enrollment) or other immunomodulating therapies (within a period of five half-lives prior to enrollment). Subjects using or intending to use prohibited concomitant medications should be excluded from enrollment in the study.

\section{Investigational Product:}

Each dapansutrile capsule consists of a blend of dapansutrile API (100 mg/capsule) and Avicel PH-101. Each Placebo Capsule contains only Avicel PH-101 and is visually identical to dapansutrile capsule.

\section{Clinical Trial Duration:}

The trial duration will be approximately 42 days for all subjects randomized, which will consist of up to 6 visits to the study site: Screening (up to 28 days prior to Baseline), Baseline (Day 1), Day 4 ( \pm 1 day), Day 8 ( \pm 2 days), Day 14 (- 4 days) and Day 28 ( \pm 2 days). Additionally, it is suggested (not required) that subjects be contacted by telephone for dosing compliance confirmation approximately every 1-2 days during the treatment period. Subjects will also receive a follow-up telephone call on Day 42 ( \pm 3 days).

\section{Safety Criteria for Evaluation:}

Safety criteria for evaluation are as follows:

- Physical examination (abbreviated general and site-specific examination)

- Vital Signs (pulse, resting blood pressure, temperature, respiration rate)

- Safety laboratory measures (chemistry, hematology and cardiac biomarkers [plasma NT-proBNP levels])

- Safety electrocardiograms (ECGs)

- Bio-impedance analysis

- Cardiopulmonary exercise test

- Transthoracic Doppler echocardiography 
Sponsor:

Olatec Therapeutics LLC
Investigational Drug:

Dapansutrile Capsules
Developmental Phase:

Phase $1 b$

- Adverse events during the clinical trial

\section{Pharmacodynamics Outcomes:}

Blood samples will be collected for PD/biomarker analysis, including:

- High-sensitivity C-reactive protein (hsCRP)

- Levels of circulating cytokines (e.g., IL-1 $\beta$, IL-6)

\section{Quality of life and Pain Questionnaire outcomes:}

Quality of life will be assessed by the Kansas City Cardiomyopathy Questionnaire and Duke Activity Status Index to measure changes in symptoms. Changes to a subject's existing aches/pains will be assessed by the Brief Pain Index.

\section{Pharmacokinetic Outcomes:}

Pharmacokinetic criteria for evaluation are as follows:

- Plasma concentrations of dapansutrile

\section{Reasons for Interruption of the Investigational Product in the Individual Subject:}

- Serious adverse event that is deemed to be possibly, probably or related to the investigational product;

- Change in systolic blood pressure, diastolic blood pressure or heart rate that exceeds $25 \%$ from baseline that is, in the opinion of the Principal Investigator, not due to another identifiable cause (e.g., non-compliance with other medications);

- New or worsening chest pain that is considered to be likely or possibly related to myocardial ischemia (angina) or elevated filling pressures (heart failure);

- New or worsening ischemic ECG changes, defined as any of the following: new or worsening ST segment deviation $>0.5 \mathrm{~mm}$ and/or T wave inversion in 2 anatomically contiguous leads;

- Abnormal cardiac rhythm including: any symptomatic ventricular or supraventricular arrhythmia; asymptomatic ventricular arrhythmia that is sustained more than 30 seconds or requires treatment; asymptomatic atrial fibrillation that is sustained more than 60 seconds; asymptomatic supraventricular tachycardia that is sustained more than 10 minutes or requires treatment; a systolic pause $>3$ seconds; a second degree type II or third degree AV block in a patient without pacemaker;

- Change in body weight that exceeds $10 \%$ from Baseline that is, in the opinion of the Principal Investigator, not due to another identifiable cause (e.g., non-compliance with other medications); 
Sponsor:

Olatec Therapeutics LLC
Investigational Drug:

Dapansutrile Capsules
Developmental Phase:

Phase $1 \mathrm{~b}$

- NYHA class IV heart failure symptoms;

- Acute infection (of any kind);

- Non-compliance with the protocol by the subject in a way that the Principal investigator is concerned about the safety of the subject and/or the accuracy of the data;

- Any new incident condition that may impair the subject to safely complete the study and/or for the Principal Investigator to accurately gather the data.

\section{Reasons for Interruption of the Study:}

- Any serious adverse event possibly, probably or related to the investigational product;

- After the first 3 or more subjects are enrolled, if: (1) 50\% or more subjects experience a serious adverse event considered unrelated to the investigational product; or (2) $75 \%$ or more subjects experience either a serious adverse event unrelated to the investigational product or a non-serious adverse event of Grade 2 or 3 intensity that is possibly, probably or related to the investigational product.

\section{Cohort Progression:}

Approximately ten (10) subjects will be randomized in the first cohort with replacement allowed for subjects who discontinue before Day 14. Upon completion of the Day 28 visit by all subjects in Cohort 1, an interim data analysis will be conducted and reviewed by the Medical Monitor, an independent cardiologist and the Principal Investigator and a decision about the progression of the study to enroll Cohort 2 will be made. This same decision process will be followed for progression from Cohort 2 to Cohort 3 except the interim data analysis will occur following the Day 8 visit by the last subject and the Day 28 visit by all other subjects in Cohort 2 .

\section{Sample Size Justification:}

An assessment of sample size or power analyses is not applicable for this Phase 1b study, as the goal of the study is to characterize any side effects following reasonable exposure to the drug.

Cohort 1 will be comprised of 8 subjects who will receive $500 \mathrm{mg}$ daily of investigational product, Cohort 2 will include 8 additional subjects who will receive $500 \mathrm{mg}$ twice daily and Cohort 3 will include 8 additional subjects who will receive $500 \mathrm{mg}$ four times daily. Each cohort will also have 2 subjects who will receive Placebo Capsules. A sample size of 24 subjects receiving dapansutrile capsules (combining Cohorts 1,2 and 3) would provide a power of $90 \%$ to exclude a difference in $>25 \%$ of any safety parameter (see Reasons for Interruption of the Investigational Product in the Individual Subject), with an estimated standard deviation of $30 \%$. 


\section{Sponsor:}

Olatec Therapeutics LLC
Investigational Drug:

Dapansutrile Capsules
Developmental Phase:

Phase $1 b$

For the pharmacodynamics analysis, the sample size of 24 subjects on investigational product would also provide a $>80 \%$ power to detect a 35\% reduction in hsCRP (e.g., from 6 to $3.9 \mathrm{mg} / \mathrm{L}$; estimated standard deviation $2 \mathrm{mg} / \mathrm{L}$ ) and a $>99 \%$ for a reduction that exceeds $50 \%$.

\section{Statistical Methods:}

Baseline measurements and demographic characteristics will be summarized using descriptive statistics with median and interquartile range for each Cohort and with data from all three cohorts combined. Descriptive summaries of categorical measurements will consist of frequencies and proportions.

The summaries for each measurement will be provided at each visit and analyzed with the nonparametric Wilcoxon or Friedman tests for paired data pooled for the 3 cohorts for 2 or more time points, respectively. The study will be underpowered to detect differences between the 3 cohorts, any comparisons between the 3 groups will be viewed as hypothesis-generating only.

Two populations will be defined for this study: 1) the Intent-to-Treat Population will consist of all subjects who have taken at least one dose of investigational product; 2) the Per-Protocol Population will consist of all subjects who take $80 \%$ or more of the total expected doses of investigational product and have no major protocol violations as determined by the Medical Monitor in consultation with the Principal Investigator.

The rationale of having double-blinded placebo allocation of 4:1 in subjects is to minimize potential biases in the subjects and study team, including site study personnel, particularly in the measures involving quality of life questionnaires and symptoms reporting. The data from the control group will be reported as the pooled data from the 3 cohorts, however because only 6 subjects will receive placebo throughout the study, comparisons with investigational product with statistical tests will not be performed.

The Statistical Analysis Plan for Study OLT1177-06 will contain a complete description of all analyses to be performed. 


\section{TABLE OF CONTENTS}

PROTOCOL APPROVAL SIGNATURE PAGE ................................................................2

PRINCIPAL INVESTIGATOR'S AGREEMENT …......................................................

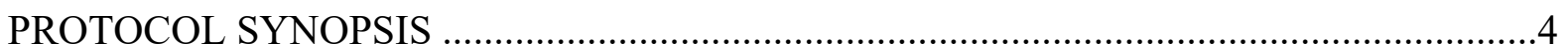

LIST OF ABBREVIATIONS AND DEFINITION OF TERMS .......................................17

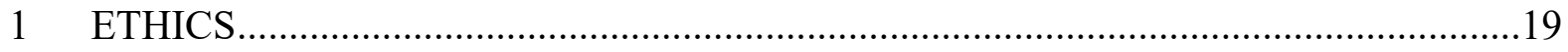

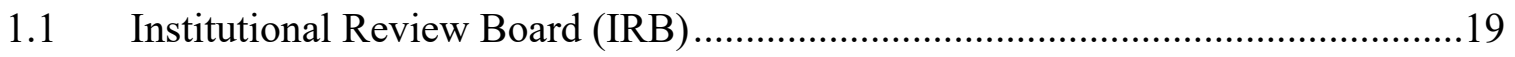

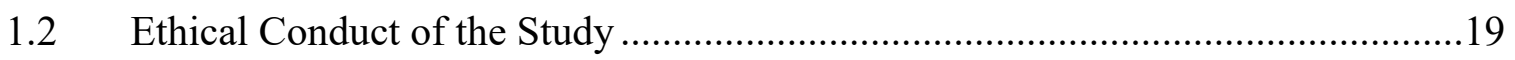

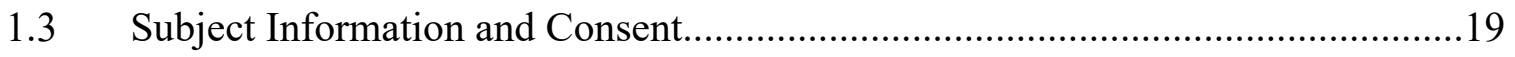

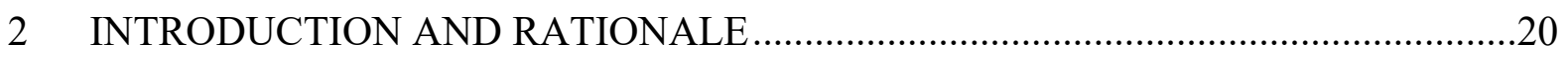

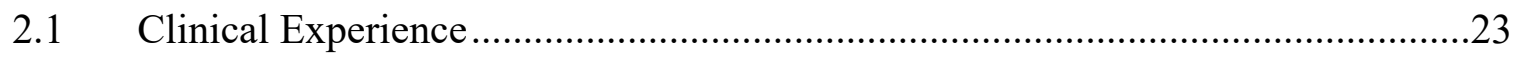

2.1.1 Study OLT1177-04 - Dapansutrile Capsules .........................................24

2.1.2 Study OLT1177-05 - Dapansutrile Capsules .............................................26

2.1.3 Studies of Dapansutrile (OLT1177) Gel..................................................28

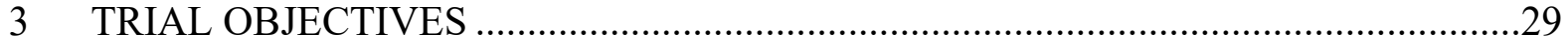

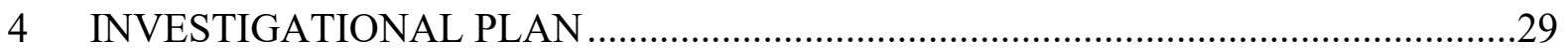

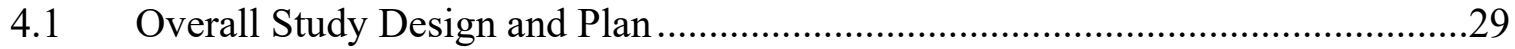

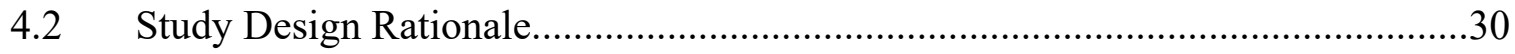

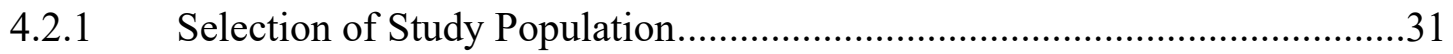

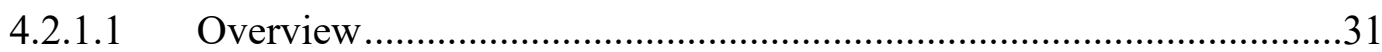

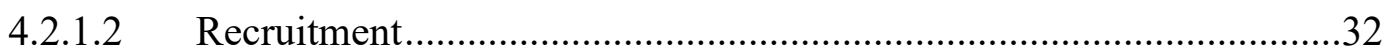

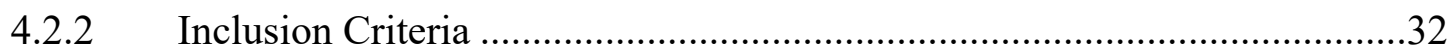

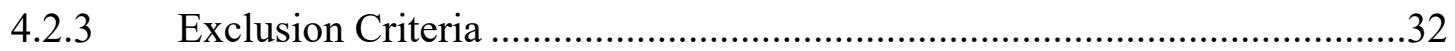

4.2.4 Early Termination of Subjects from Therapy ..........................................

4.2.4.1 Subject-Specific Stopping Rules....................................................35

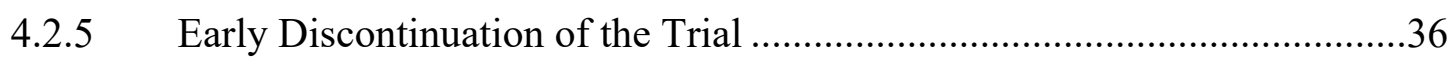

4.2.5.1 Study-Level Stopping Rules …….....................................................

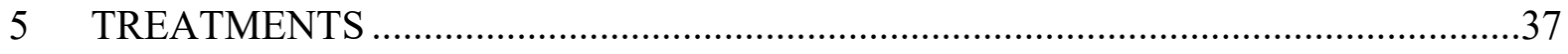

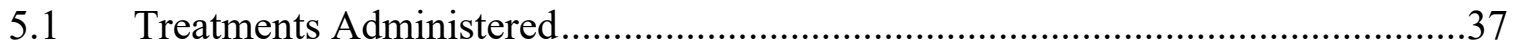

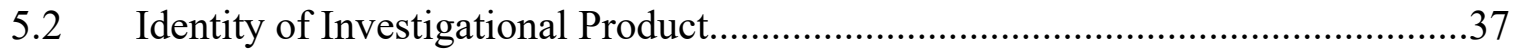

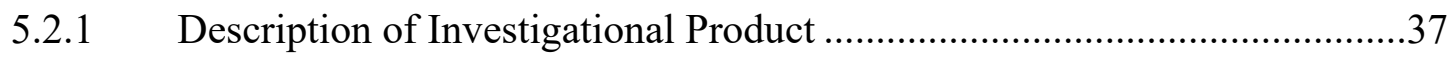

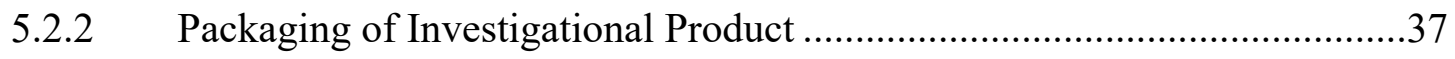

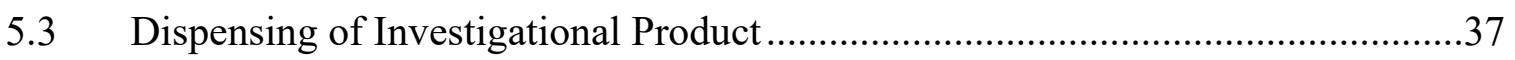

5.4 Method of Assigning Subjects to Treatment Groups..........................................37

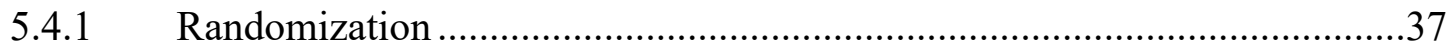




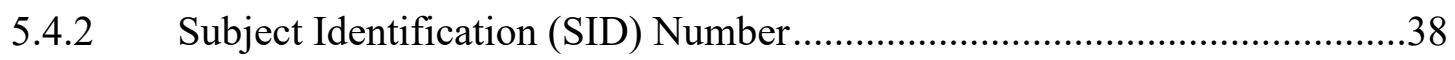

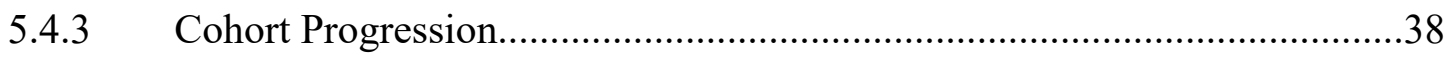

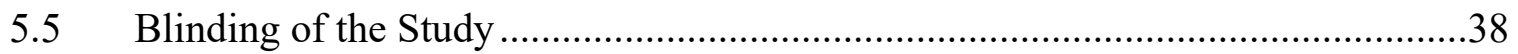

5.6 Prior and Concomitant Medications/Therapies .......................................................39

5.6.1 Allowed Concomitant Medications/Therapies ………………….................39

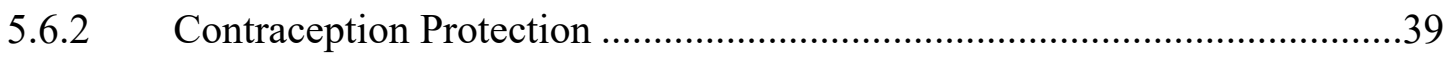

5.6.3 Prohibited Concomitant Medications/Therapies...........................................39

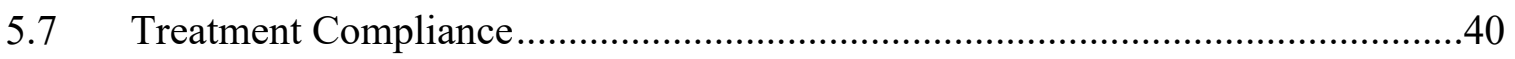

5.8 Investigational Product Storage \& Accountability ...............................................41

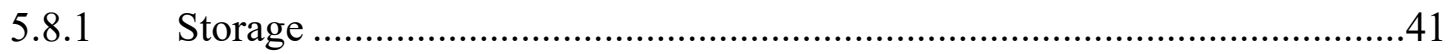

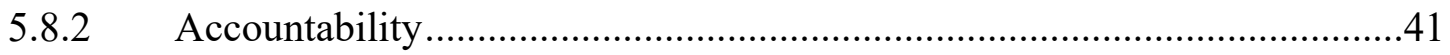

6 SAFETY, PHARMACODYNAMICS AND DISEASE-STATE VARIABLES .............42

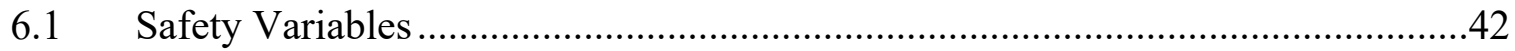

6.2 Pharmacodynamics / Biomarker Variables........................................................42

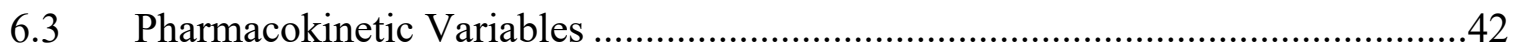

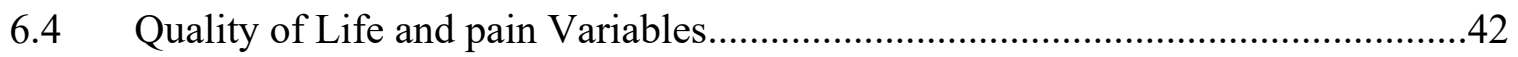

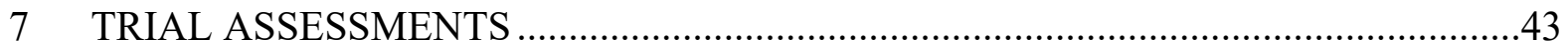

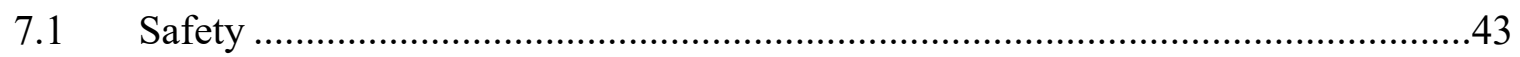

7.1.1 Medical History and Concomitant Medications/Therapies Review ............43

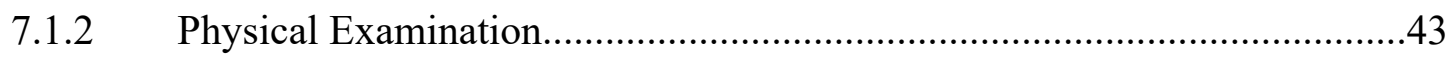

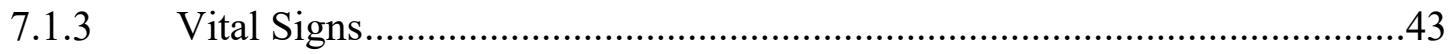

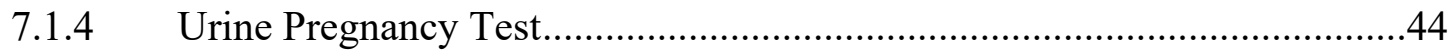

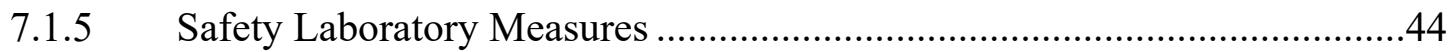

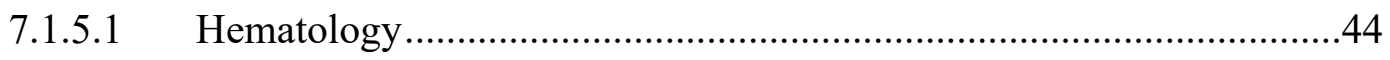

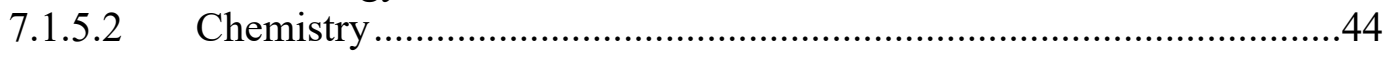

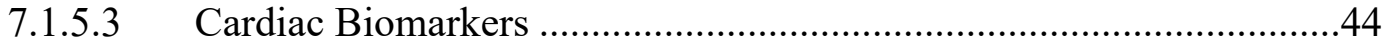

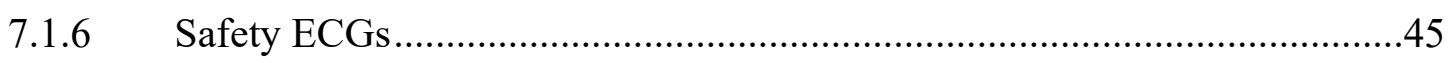

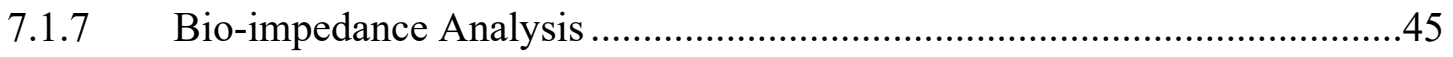

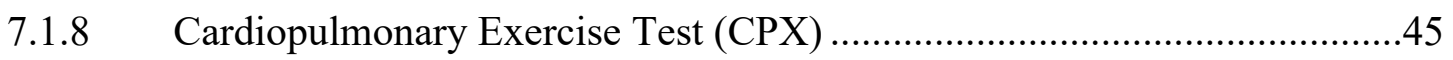

7.1.9 Transthoracic Doppler Echocardiography ...................................................46

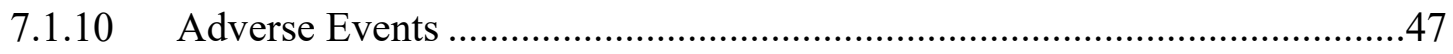

7.2 Pharmacokinetic \& Pharmacodynamics Assessments.............................................47

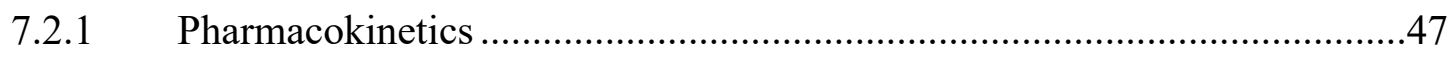

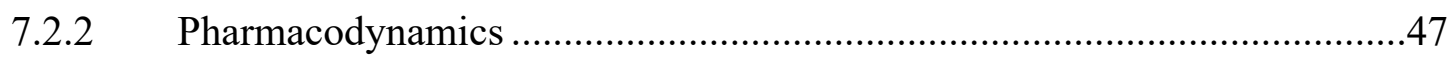

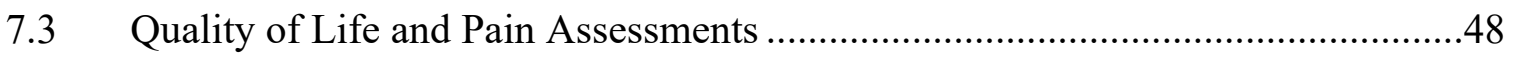

7.3.1 Kansas City Cardiomyopathy Questionnaire (KCCQ) ................................48 


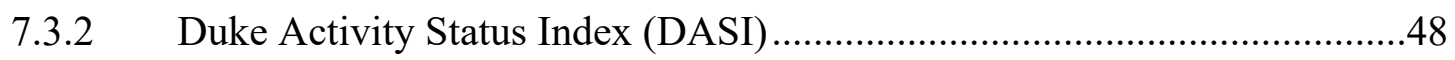

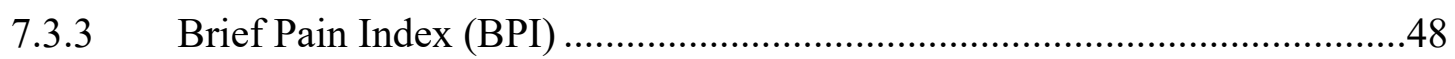

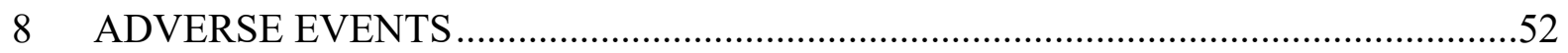

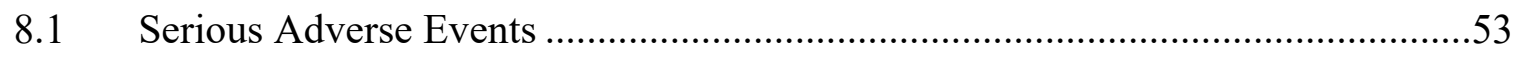

$8.2 \quad$ Unexpected Adverse Event.......................................................................54

8.3 Suspected Unexpected Serious Adverse reactions (SUSARs) ...............................54

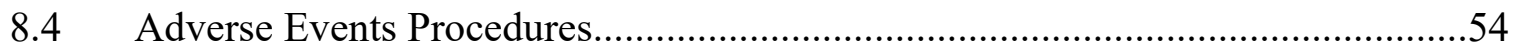

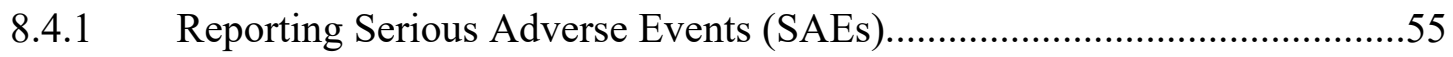

8.4.1.1 Reporting SAEs to the Sponsor ..........................................................5

8.4.1.2 Contact Information for Reporting Serious Adverse Event...................55

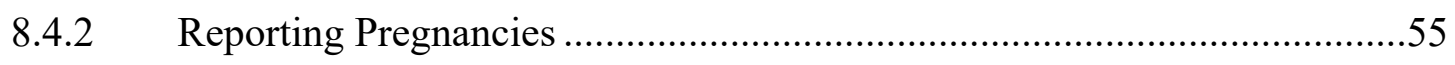

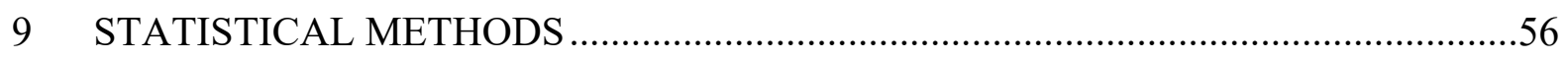

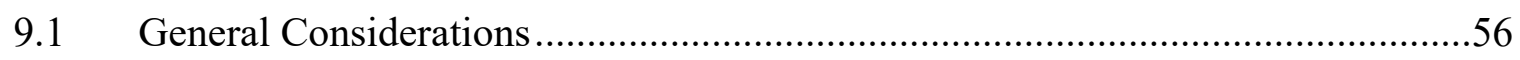

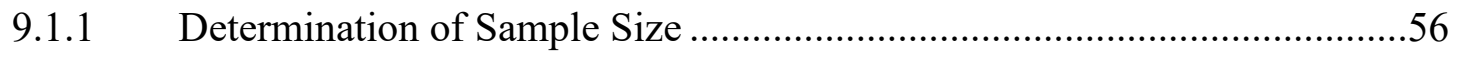

9.1.2 Statistical and Analytical Plans.............................................................56

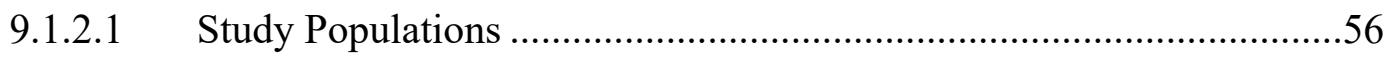

9.1.2.2 Planned Statistical Analyses ………………………………………....56

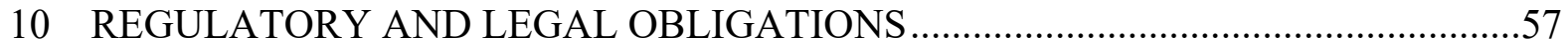

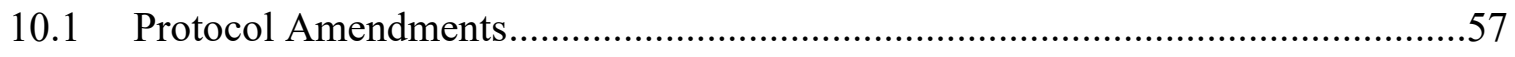

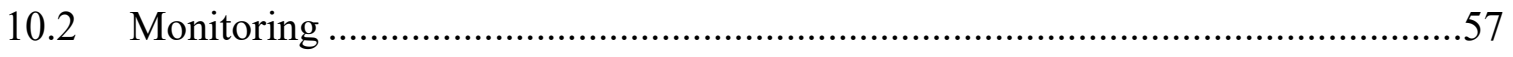

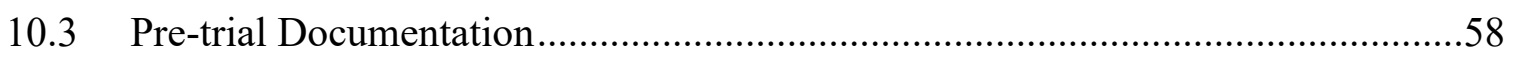

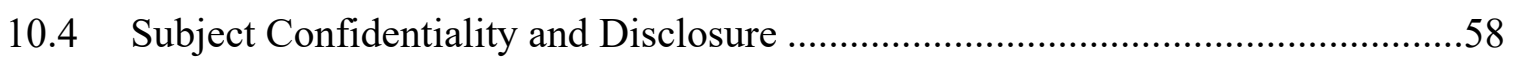

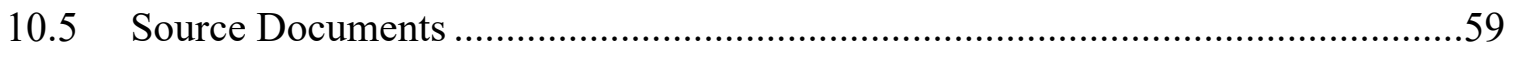

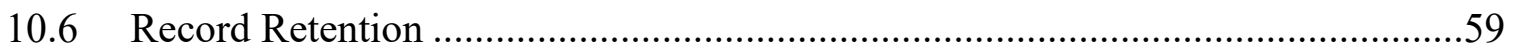

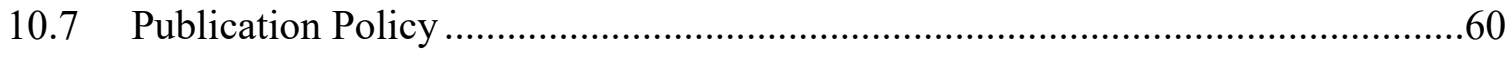

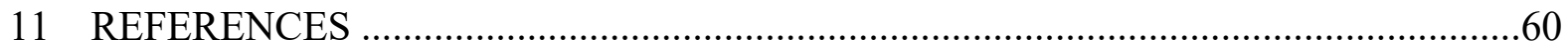




\section{LIST OF IN-TEXT TABLES}

Table 5.1 Prohibited Concomitant Medications/Therapies..........................................40

Table 7.1 Schedule of Trial Activities \& Assessments..................................................50

Table 8.1 Classification of Adverse Events by Intensity ..........................................52

Table 8.2 Guidelines for Determining the Relationship Between Any Adverse Event and the Investigational Product ......................................................53

\section{LIST OF APPENDICES}

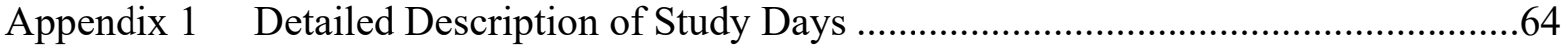

Appendix 2 American Heart Association Guidelines for Collection of Blood Pressure.....70

Appendix 3 Contact List....................................................................................... 71 


\section{LIST OF ABBREVIATIONS AND DEFINITION OF TERMS}

\begin{tabular}{|c|c|}
\hline Abbreviation & Definition \\
\hline ACR & American College of Rheumatology \\
\hline $\mathrm{AE}$ & Adverse Event \\
\hline ALT & Alanine transaminase (SGPT) \\
\hline API & Active pharmaceutical ingredient \\
\hline ASA & Acetylsalicylic acid (aspirin) \\
\hline AST & Aspartate transaminase (SGOT) \\
\hline AUC & Area under the concentration-time curve \\
\hline BID & Twice a day \\
\hline BUN & Blood urea nitrogen \\
\hline $\mathrm{CBC}$ & Complete Blood Count \\
\hline COX-1 & Cyclooxygenase-1 \\
\hline $\mathrm{COX}-2$ & Cyclooxygenase- 2 \\
\hline $\mathrm{cm}$ & Centimeter \\
\hline $\mathrm{C}_{\max }$ & Maximum observed plasma concentration \\
\hline $\mathrm{CRF}$ & Case report form \\
\hline $\mathrm{CRO}$ & Contract Research Organization \\
\hline CSF & Colony stimulating factor \\
\hline DAAAP & Division of Anesthesia, Analgesia and Addiction Products, CDR, FDA \\
\hline ECG & Electrocardiogram \\
\hline EMA & European Medicines Agency \\
\hline FDA & Food and Drug Administration \\
\hline $\mathrm{g}$ & Gram \\
\hline GCP & Good Clinical Practice \\
\hline GGT & Gamma-glutamyl transferase \\
\hline $\mathrm{HbAlc}$ & Glycated haemoglobin \\
\hline $\mathrm{HBsAg}$ & Hepatitis B surface antigen \\
\hline $\mathrm{HCV}$ & Hepatitis $\mathrm{C}$ virus \\
\hline hsCRP & High sensitivity C-reactive protein \\
\hline ICF & Informed consent form \\
\hline $\mathrm{ICH}$ & International Conference on Harmonisation \\
\hline IRB & Institutional Review Board \\
\hline
\end{tabular}




\begin{tabular}{|c|c|}
\hline Abbreviation & Definition \\
\hline IL & Interleukin \\
\hline $\mathrm{kg}$ & Kilogram \\
\hline $\mathrm{mg}$ & Milligram \\
\hline $\mathrm{mL}$ & Milliliter \\
\hline ng & Nanogram \\
\hline NLRP3 & NOD-like receptor, pyrin domain containing 3 \\
\hline NSAIDs & Nonsteroidal anti-inflammatory drugs \\
\hline NYHA & New York Heart Association \\
\hline OTC & Over-the-counter \\
\hline QT & QT interval \\
\hline QTcF & QT interval with correction by Fridericia's formula \\
\hline QD & Once a day \\
\hline PK & Pharmacokinetic \\
\hline PPP & Per-protocol Population \\
\hline PRN & As needed \\
\hline RBC & Red blood cell \\
\hline SAE & Serious adverse event \\
\hline SID & Subject identification number \\
\hline SUSAR & Suspected Unexpected Serious Adverse Reaction \\
\hline TEAE & Treatment-emergent adverse event \\
\hline TCAs & Tricyclic antidepressants \\
\hline TID & Three times a day \\
\hline TNF & Tumor necrosis factor \\
\hline
\end{tabular}




\section{ETHICS}

\subsection{INSTITUTIONAL REVIEW BOARD (IRB)}

The protocol, protocol amendments, subject recruiting materials, the informed consent form and any other materials provided to subjects must be approved by the Western Institutional Review Board (Olympia, WA) operating in compliance with 21 CFR Part 56. A copy of the approval letter must be received by the Sponsor and/or CRO prior to shipment of drug supplies to the site.

Records of the Institutional Review Board's review and approval of all documents pertaining to the study must be kept on file by the investigator and are subject to sponsor and FDA inspection at any time. The Investigator will provide required progress reports and report all SAEs to the IRB.

\subsection{ETHICAL CONDUCT OF THE STUDY}

This trial will be performed in accordance with ethical principles that have their origin in the Declaration of Helsinki and conducted in adherence to the trial protocol, Good Clinical Practices (GCPs) as defined in Title 21 of the US CFR Parts 50, 54, 56, 312 and Part 11 as well as ICH GCP consolidated guidelines (E6) and applicable regulatory requirements.

\subsection{SUBJECT INFORMATION AND CONSENT}

Informed consent will be obtained from all subjects prior to any trial procedures being performed. All subjects will be given ample time to review the informed consent form and ask questions. A signed ICF must then be obtained prior to any trial procedures being performed. 


\section{INTRODUCTION AND RATIONALE}

There is a clear pathophysiologic link between inflammation and cardiovascular diseases (Abbate 2012, Libby 2005, Mason 2015, Seropian 2014, Toldo 2015, Van Tassell 2013a). Pro-inflammatory cytokines, like IL-1 $\beta$, promote atherosclerosis, thrombosis, acute myocardial infarction (AMI) and HF (Dinarello 2011, Van Tassell 2013a). IL-1 $\beta$ activity is tightly regulated at multiple steps. Pro-IL-1 $\beta$ is synthetized in response to inflammatory stimuli such as pathogen-associated molecular patterns and danger-associated molecular patterns, and yet it requires activation by cleavage within a macromolecular structure known as the inflammasome. The inflammasome is characterized by a scaffold protein, ASC (or apoptosis speck-like protein containing a caspase recruiting domain) and a sensor protein, part of the nucleotide-binding and oligomerization domain (NOD)-like receptors (NLR). The NLR pyrin domain containing 3 (NLRP3) also known as cryopyrin is the most widely characterized inflammasome sensor in the pathophysiology of cardiovascular disease (Latz 2013, Toldo 2015).

NLRP3 resides within the cells and senses perturbations of cell homeostasis as well as intracellular danger in response to infectious stimuli or cellular stress. NLRP3 activation leads to recruitment of ASC, which consists of two death-fold domains: one pyrin domain and one caspase activation and recruitment domain (CARD). ASC interacts with NLRP3 via the pyrin domain, which promotes assembly of the inflammasome. Using its CARD, ASC brings monomers of pro-caspase-1 into proximity fostering self-cleavage and formation of active caspase-1. Following inflammasome activation, caspase-1 proteolytically cleaves precursors of IL-1 $\beta$ and IL-18 into their biologically active forms, and facilitates its shuttling across the plasma membrane. Once released, IL-1 $\beta$ binds to the IL-1 receptor (IL-1R) expressed on several cell types, leading to downstream signaling and a cascade of inflammation involving other pro-inflammatory cytokines, chemokines and several other mediators (Dinarello 2011).

IL-1 $\beta$ was identified as a soluble cardiodepressant factor in subjects with sepsis in 1996 (Kumar 1996). More recently, IL-1 $\beta$ has been identified as a cause of reversible cardiac dysfunction in HF. Plasma derived from HF subjects induced a reversible cardiac dysfunction (i.e., left ventricular systolic dysfunction and impaired contractile reserve) in mice, which was preventable by pretreating with recombinant human IL-1R antagonist, anakinra (Van Tassell 2012). Further, a single intraperitoneal (IP) injection of IL-1 $\beta$ (3 $\mu \mathrm{g} / \mathrm{kg})$ induced systolic dysfunction and impaired response to isoproterenol in mice, and repeated injections of IL-1 $\beta$ gave a reversible nonischemic cardiomyopathy (Van Tassell 2013b). Using this model, Toldo et al (2014) showed that blockade of IL-18 prevented IL-1 $\beta$-induced dysfunction, but not vice versa, since IL-1 $\beta$ blockade with anakinra failed to prevent IL-18-induced dysfunction. 
These results are supported by findings that processing of IL- $1 \beta$ via the NLRP 3 inflammasome and the downstream signaling via IL-1R is associated with the development of coronary heart disease, myocardial remodeling and HF. The hearts of transgenic mice deficient in ASC and caspase-1 or NLRP3 showed a marked improvement in cardiac healing and function compared to the hearts of wild-type mice after ischemia/reperfusion (Kawaguchi 2011, Mezzaroma 2011, Sandanger 2013) and pharmacologic inhibition of the NLRP3 inflammasome preserves cardiac function after AMI in mice (Marchetti 2014, Marchetti 2015). Disruption of IL-1 signaling in infarcted IL-1R-deficient mice was cardioprotective and favored remodeling post-AMI (Bujak 2008, Abbate 2011). Moreover, IL-1 $\beta$ blockade with anakinra, recombinant IL-1 $\beta$ receptor antagonist, or with an IL-1 $\beta$ antibody significantly ameliorates the remodeling process by inhibiting cardiomyocyte apoptosis in experimental AMI murine models (Abbate 2008, Toldo 2013, Harouki 2017). Phase 2 clinical trials in subjects with ST-segment elevation AMI have shown an overall favorable safety profile of anakinra and a signal for reduced incidence of HF (Abbate 2010, Abbate 2013, Abbate 2015) and in proof-of-concept studies of subjects with symptomatic heart failure, anakinra appears also to be well tolerated and associated with signals of improved exercise capacity (Van Tassell 2012, Van Tassell 2016, Van Tassell 2017).

Dapansutrile (3-methanesulfonyl-propionitrile) is a small molecule that has been shown to modulate the activity of pro-inflammatory cytokines IL-1 $\beta$ and IL-18 via inhibition of the NLRP3 inflammasome. In human monocyte-derived macrophages, $1 \mu \mathrm{M}$ dapansutrile significantly decreased release of IL-1 $\beta$ by $60 \%$ and IL-18 by $70 \%$ (Marchetti 2018). Mechanistic studies have been carried out and confirm that dapansutrile prevents formation of the NLRP3 inflammasome (Marchetti 2018). Specifically, corrected Fluorescent Resonance Energy Transfer (FRETc) analysis showed that dapansutrile limited NLRP3-ASC and NLRP3caspase-1 interaction, thus preventing inflammasome oligomerization (Marchetti 2018). FRET positivity requires the distance between two molecules, in this case NLRP3 and ASC, to be less than 30 nanometers. The central domain of NLRP3 exhibits nucleotide-binding properties associated with ATPase activity, which is required for the NLRP3 selfoligomerization and ASC recruitment during inflammasome formation (Duncan 2007). Dapansutrile was shown to reduce ATPase activity suggesting a direct interaction with the NLRP3 protein, an effect that has been demonstrated by other inflammasome inhibitors (Juliana 2010, He 2014).

Due to its mechanism of action, dapansutrile may be an efficacious IL-1 $\beta$-modulating therapy to treat inflammation in subjects with cardiovascular disease. In support of this hypothesis, dapansutrile was studied in an experimental mouse model of AMI due to ischemia reperfusion injury (Toldo 2017, Toldo 2019). Mice treated with dapansutrile $(6,60$ or $600 \mathrm{mg} / \mathrm{kg}$ intraperitoneal dose) were shown to have reduced infarct size and improved left ventricle function compared to the vehicle-treated group. Specifically, experimental AMI was induced 
in adult male mice by ligation of the proximal left coronary artery for 30 minutes followed by release and reperfusion as described previously (Toldo 2011, Abbate 2008).

OLT1177 treatment at reperfusion showed significant dose-dependent reduction in infarct size $(-36 \%,-67 \%$, and $-62 \%$ for 6,60 , and $600 \mathrm{mg} / \mathrm{kg}$, respectively; $\mathrm{P}<0.001$ for linear trend, $\mathrm{P}=0.010$ vs. vehicle for $6 \mathrm{mg} / \mathrm{kg}$, and $\mathrm{P}<0.001$ vs. vehicle for 60 and $600 \mathrm{mg} / \mathrm{kg}$ ). Cardiac systolic function as left ventricular fractional shortening was preserved at 24 hours and 7 days after injury ( $\mathrm{P}=0.015$ for $6 \mathrm{mg} / \mathrm{kg}$ and $\mathrm{P}<0.01$ for 60 and $600 \mathrm{mg} / \mathrm{kg}$ ). OLT1177 reduced infarct size also when given after 60 minutes of reperfusion $(271 \%, \mathrm{P}<0.001$ vs. vehicle). (Toldo 2019).

A comprehensive nonclinical safety evaluation program has been conducted to support the clinical development of dapansutrile as both a topical and oral formulation, and Olatec has four (4) open INDs at FDA. Under IND 106876, dapansutrile gel for the topical route of administration is being studied in subjects with inflammation associated with chronic musculoskeletal pain, such as osteoarthritis of the knee. In parallel to dapansutrile gel development, dapansutrile capsules are being studied by oral administration under IND 122165 for the treatment of pain due to musculoskeletal conditions (e.g., gouty arthritis). Dapansutrile capsules are also under investigation for the treatment of the rare disease Schnitzler's syndrome (IND 137829, Division of Pulmonary, Allergy, and Rheumatology Products) and for evaluation in cardiovascular disease indications (IND 136589, Division of Cardiovascular and Renal Products).

Dapansutrile was well tolerated in dermal and oral toxicity studies in rats and dogs, with no significant target organ toxicity. Dapansutrile was not mutagenic in any genotoxicity assay, and dapansutrile gel was not irritating to rabbit skin or eyes and was not a skin sensitizer. When dapansutrile was administered orally to rats, no specific target tissue or organ system was identified in a 6-month repeat-dose pivotal toxicity study. In a repeat-dose toxicity study in dogs dosed once daily for 30 days via oral gavage, clinical observations of dark colored urine were observed in males at the high dose $(750 \mathrm{mg} / \mathrm{kg})$. In addition, dose-dependent gross and microscopic findings in the urinary bladder, including gross distension, bladder thickening, red foci, and microscopic edema (ranging from minimal to moderate) and hemorrhage (ranging from minimal to mild) within the bladder wall were observed. Results of safety pharmacology studies indicate that the respiratory and cardiovascular systems are target organ systems in these species. Decreased body temperature and respiratory rates were observed in rats at oral doses of 1,000 and 2,000 mg/kg, or human equivalent doses of 161 and $323 \mathrm{mg} / \mathrm{kg}$. The effects were mild to moderate in degree. There were slight dose-dependent effects on heart rate and blood pressure in the dog, which were transient and fully reversible. The maximum oral dose qualified in nonclinical studies is determined from the dog 30-day oral no observable adverse effect level (NOAEL) of $100 \mathrm{mg} / \mathrm{kg}$, which translates into a human equivalent dose of $55.6 \mathrm{mg} / \mathrm{kg}$ per day, or $3.3 \mathrm{~g} /$ day in a $60-\mathrm{kg}$ human. 
In human subjects, dapansutrile has been shown to be safe and well tolerated in three completed clinical studies of up to 6 weeks in duration by the topical route of administration and two completed studies of up to 8 days duration by oral administration (at oral doses up to $2,000 \mathrm{mg} /$ day). In these trials, a total of 185 and 63 subjects have been exposed to dapansutrile gel and dapansutrile capsules, respectively, with two treatment-emergent serious adverse events reported, neither of which was considered related to dapansutrile, and no deaths have been reported with exposure.

Olatec is proposing to conduct a study of dapansutrile capsules in clinically stable subjects with chronic symptomatic systolic HF (New York Heart Association [NYHA] functional classification II-III) to better characterize the safety of dapansutrile in subjects with HF that are vulnerable to potential side-effects of anti-inflammatory drugs. Based on its mechanism of action, dapansutrile is not expected to inhibit the basal production of prostaglandins and thus not affect blood pressure, renal perfusion and filtration, or gastric sodium and water clearance. Dapansutrile is not expected to have the unwanted action as nonsteroidal antiinflammatory drugs (NSAIDs), cyclooxygenase-2 (COX-2) inhibitors or corticosteroids. Prior experience with IL-1 blockers, anakinra and canakinumab, show no effects on blood pressure, renal perfusion and filtration, or gastric sodium and water clearance.

\subsection{CLINICAL EXPERIENCE}

Two clinical studies have been completed with dapansutrile capsules: Study OLT1177-04 and Study OLT1177-05. Study OLT1177-04 was a Phase 1 single- and multi-dose safety and pharmacokinetic study of orally administered dapansutrile capsules for up to eight consecutive days at doses ranging from $100 \mathrm{mg}$ QD to 1,000 $\mathrm{mg}$ QD in normal healthy volunteers and was conducted in a single clinical site in the United States. Study OLT1177-05 was an open-label Phase 2a, single-center, sequential, result-dependent dose adaptation proof-of-concept safety and efficacy study in subjects with an acute gout flare with daily doses ranging from $100 \mathrm{mg}$ to $2000 \mathrm{mg}$ (500 $\mathrm{mg}$ QID).

Additionally, three clinical studies have been completed with the investigational product in a topical dosage form (dapansutrile gel), consisting of a Phase 1 topical dose-escalation safety study in normal healthy subjects (Study OLT1177-01), a Phase 2a 14-day topical safety and efficacy study (Study OLT1177-02) in subjects with mild-to-moderate osteoarthritis of the knee and a Phase 2b 6-week topical efficacy and safety study (Study OLT1177-03) in subjects with mild-to-moderate osteoarthritis of the knee. These studies were conducted at multiple clinical sites in the United States.

A summary of the known and potential risks and benefits, if any, to human subjects is contained in the Investigators Brochure for dapansutrile. 


\subsubsection{Study OLT1177-04 - Dapansutrile Capsules}

Study OLT1177-04 was a Phase 1 single-center, placebo-controlled, sequential group, doseescalation study of the safety and PK of 3 dosages of dapansutrile capsules in 35 healthy male and female subjects aged 18 to 60 years. Dapansutrile capsules were administered orally at doses of 100, 300 and 1,000 mg in single-dose fasted (Part A), single-dose fed (Part B, $1,000 \mathrm{mg}$ dose only) and multiple-dose (Part C) regimens. In Part A, 18 healthy volunteer subjects in 3 dose escalating cohorts were randomized to receive a single dose of the investigational product ( 5 subjects dapansutrile capsules and 1 subject Placebo Capsules in each cohort). Upon completion of Part A, subjects from the high dose group (1,000 mg) received a high fat meal and single 1,000 mg dose of dapansutrile capsules in Part B of the study. Upon completion of Part B, a new subject population of 17 subjects in 3 dose escalating cohorts was randomized in Part $\mathrm{C}$ of the study. Part $\mathrm{C}$ subjects received a total of 8 doses over 8 consecutive days given once per day and were followed for up to 30 days for safety assessment.

The primary objective of the study was to assess the safety and tolerability of dapansutrile capsules in healthy subjects after oral administration. Secondary objectives were to: characterize and compare the pharmacokinetic profile of dapansutrile following single ascending doses by oral administration; evaluate the effect of food on the single-dose pharmacokinetics of dapansutrile; and characterize and compare the multiple-dose PK profiles of dapansutrile following 8 consecutive days of oral administration.

Safety and tolerability was assessed by physical examination and measurement of vital signs, ECGs and clinical laboratory tests (e.g., chemistry, hematology, lipid profile, coagulation factor and urinalysis). Further, adverse events and concomitant medications were recorded. Blood was drawn for plasma dapansutrile PK analysis at pre-dose, 30 minutes and 1, 1.5, 2, 2.5, 3, 3.5, 4, 4.5, 5, 6, 8, 10, 12, 23, 48, 72, 96 and 168 hours post-dose in Part A. For Part B, blood was drawn prior to dosing and after 30 minutes and 1, 1.5, 2, 2.5, 3, 3.5, 4, 4.5, 5, 6, 8, $10,12,23,48,72,96$ and 168 hours post-dose. For Part C, blood was drawn prior to dosing and 1, 2, 3, 3.5, 4, 4.5, 5, 6, 8, 10, 12 and 23 hours post-dose on Day 1, pre-dose on Days 6 and 7; and on Day 8 prior to dosing and after 1, 2, 3, 3.5, 4, 4.5, 5, 6, 8, 10, 12 and 23 hours postdose.

A total of 35 subjects were randomized in the study. Five subjects in each of the 3 active treatment groups and 1 in each of the 3 placebo groups in Parts A and $\mathrm{C}$ of the study were enrolled, with the exception of Cohort 5 for which only 4 subjects were enrolled. All enrolled subjects completed the study, with no discontinuations, and safety data were collected for all subjects enrolled. PK data were collected and reported only for subjects in the active treatment groups. 
No deaths or serious adverse events occurred during the study. A total of 7 out of 35 (20.0\%) subjects reported a total of 7 treatment-emergent adverse events with six occurring in the dapansutrile capsules treatment group and one occurring in the Placebo Capsules group. The observed TEAEs in the dapansutrile capsules treatment group were one instance each of: diarrhea, back pain, migraine, contact dermatitis, eczema and headache. Five of the seven events occurred in Parts A and B of the study, and two occurred in Part C (both in the low dose group). None of the events were considered to be drug-related and all resolved. The severities of the events ranged from mild to moderate.

Results from all the clinical laboratory evaluations were variable, but showed no relevant changes with regard to the change from baseline or study drug administration timing or dose or between the active treatment groups and placebo. ECG findings were either normal or not considered to be clinically significant. QT and QTcF durations remained stable throughout the study in all parts of the study. Respiration rate remained largely consistent throughout the study.

Overall, dapansutrile capsules were found to be safe and well tolerated in healthy subjects. No treatment-emergent serious adverse events or deaths occurred. The few adverse events that did occur were mild to moderate in severity, deemed unrelated to the study drug, and all resolved. None of the laboratory parameters, or cardiac or respiratory measures indicated any issues with the drug.

Single- and multiple-dose PK profiles were determined as a secondary endpoint of Study OLT1177-04. After a single oral dose of 100,300 or 1,000 mg dapansutrile capsules to fasted healthy subjects, dapansutrile was rapidly absorbed with mean peak plasma concentrations of 2,700, 9,800 and 32,000 ng/mL, respectively, reached at approximately 1.8 hours. Plasma dapansutrile concentrations were detectable in all subjects in all dapansutrile-dosed treatment groups at all time points. Seven days after dapansutrile capsules administration, plasma dapansutrile concentrations were still detectable for all subjects but mean concentrations had fallen to $14.8,69.9$ and $270 \mathrm{ng} / \mathrm{mL}$ at the 168 hour time point for 100 , 300 and 1,000 mg dapansutrile doses. Systemic exposure (AUC and $\mathrm{C}_{\max }$ ) increased linearly with dose. The median terminal half-life ranged from 21 to 24 hours for all dose groups.

For characterization of multi-dose $\mathrm{PK}$ in Part $\mathrm{C}$, dapansutrile capsules were administered once daily over the course of 8 days to 3 cohorts in a sequential dose-escalation design. Doses of 100,300 or $1,000 \mathrm{mg}$ were administered orally. Blood samples were collected for PK analysis at the following time points: prior to dosing and 1, 2, 3, 3.5, 4, 4.5, 5, 6, 8, 10, 12 and 23 hours post-dose on Day 1, pre-dose on Days 6 and 7; and on Day 8 prior to dosing and after 1, 2, 3, $3.5,4,4.5,5,6,8,10,12$ and 23 hours post-dose. 
After 8 daily doses of dapansutrile capsules over 8 days, the mean maximum plasma dapansutrile concentrations were reached 1 to 2 hours after the final dose was administered for all dose groups. The mean $\mathrm{C}_{\max }$ increased linearly with dose for the groups receiving 100, 300 or $1,000 \mathrm{mg}$ dapansutrile per day $(4,800,15,800$ and $41,400 \mathrm{ng} / \mathrm{mL}$, respectively). Plasma concentrations on Day 6 and 7 and pre-dose on Day 8 corresponded to trough levels of $1,400-1,900,6,600-7,300$ and 1,900-19,500 $\mathrm{ng} / \mathrm{mL}$ for the low, mid and high dose groups, respectively. Systemic dapansutrile exposure following the last dose (AUC) also increased linearly with dose. The median terminal half-life ranged from 14 to 21 hours for all dose groups.

Overall, dapansutrile capsules were found to be safe and well tolerated in healthy subjects and Study OLT1177-04 is supportive of continued research with the dapansutrile investigational product.

\subsubsection{Study OLT1177-05 - Dapansutrile Capsules}

Study OLT1177-05 is an open-label Phase 2a, single-center, sequential, result-dependent dose adaptation proof-of-concept study in subjects with an acute gout flare. The objective of the study was to evaluate the safety profile, clinical activity and changes in the inflammatory biomarkers of oral dapansutrile capsules. The study was designed to evaluate four different dapansutrile doses in four cohorts (each approximately 8 subjects) to support future clinical investigation of oral dapansutrile capsules for the treatment of acute gout flare. The study has completed and a total of 34 subjects were enrolled and 30 completed the study. Dapansutrile capsules were safe and well tolerated at all doses. There were no pathologic changes in metabolic, physiological or hematological measurements for any dose group.

Safety assessments were conducted at each visit and included monitoring the occurrence of adverse events (AEs), changes in abbreviated physical examination findings, vital signs and clinical safety laboratory test results (chemistry, hematology and urinalysis).

In this open-label study, based on audited preliminary data, a total of 25 out of 34 (73.5\%) subjects reported a total of 45 TEAEs. The majority of AEs were metabolism and nutrition disorders (37.8\%) and gastrointestinal disorders (22.2\%). Of 17 metabolism and nutrition disorders, all but two (decreased appetite and hyponatremia) were instances of gout or gout flare. Gastrointestinal AEs included one instance of constipation, three of diarrhea, three of flatulence, two of abnormal borborygmi (gastrointestinal sounds), and one instance of nausea. The severity of events ranged from mild to severe. Two AEs (flatulence and headache) reported in one subject were considered probably related to dapansutrile capsules and 7 AEs of gout, reported in 6 subjects, were considered as possibly related, as were 2 AEs each of diarrhea, flatulence and abnormal borborygmi, and one $\mathrm{AE}$ each of headache, dysgeusia, rhinorrhoea, hyperhidrosis and constipation. 
No deaths occurred during the study. Two subjects, both in Cohort 1, reported serious adverse events during the study. The first subject was a 68-year-old male subject (Subject \#032-002) who received four doses of dapansutrile Capsules ( 5 x $100 \mathrm{mg}$ capsules per dose) over 3 days before he stopped therapy early. Two days after the last dose of investigational product, the subject was hospitalized due to worsening gout flare. He was treated with diclofenac $100 \mathrm{mg}$ orally twice a day for four days and methylprednisolone $500 \mathrm{mg}$ intravenously (IP) once a day for three days. The subject's medical history is relevant for tophaceous gout, hypertension and prostatic hypertrophy. Concomitant medications taken within 2 weeks of the SAE included allopurinol $300 \mathrm{mg}$ orally (PO) QD, prednisone $30 \mathrm{mg}$ PO QD, nifedipine $30 \mathrm{mg}$ PO QD, and pantoprazole $20 \mathrm{mg}$ PO QD. The suspected cause of this SAE was the initiation of allopurinol therapy 3 weeks prior to enrollment, which was a major protocol deviation (i.e., allopurinol was prohibited unless the subject was on a stable dose for at least 3 months prior to the baseline visit). In the Principal Investigator's opinion, the gout flare was of moderate severity, unrelated to the investigational product and unexpected. The event resolved without sequelae 3 days after it began. Investigational Medicinal Product (IMP) was discontinued due to the SAE.

On 09 February 2018, 18 days after the last dose of OLT1177 Capsules, the second subject was admitted in the hospital due to chest pain after an elective percutaneous coronary intervention (PCI). The chest pain he experienced was attributed to post-PCI myocardial infarction due to $99 \%$ stenosis of the D1 branch of the left coronary artery. During admission his ECG showed several times that he had non-sustained ventricular tachycardia and he remained in the hospital for observation. He was started on treatment with spironolactone and acetylsalicylic acid. His cardiac rhythm returned to normal and the cardiac symptoms resolved. The event was considered resolved, and he was discharged from the hospital, on 14 February 2018. Spironolactone and acetylsalicylic acid were continued after discharge. During hospitalization he had a gout flare in the right first metatarsophalangeal joint, which started on 13 February 2018. On evaluation it was found to be mild in severity and was not considered an SAE. He was started on treatment with prednisolone. The gout flare was not resolved at the time of hospital discharge and prednisolone was continued. In the Principal Investigator's opinion, the post-PCI infarction was considered moderate in severity, unrelated to the IMP, and unexpected. No action was taken with the IMP in response to the event.

Efficacy outcomes were evaluated by subject-reported pain and disability scales, Global Evaluation of Treatment, Investigator-assessed Index Joint Score and Global Rating of Disease, and analysis of biomarkers of inflammation. Study OLT1177-05 has completed and the data are being analyzed. 


\subsubsection{Studies of Dapansutrile (OLT1177) Gel}

Three studies have been conducted with the topical formulation of dapansutrile, dapansutrile gel. In total, 185 subjects were treated with dapansutrile gel at doses up to $6 \mathrm{~mL}$ TID.

No treatment-emergent serious adverse events or deaths occurred in any of the dapansutrile gel studies. Across all studies, adverse events were mild to moderate in severity, and included specifically: sinus infection, toothache, cold, migraine, neck pain, insomnia, pharyngitis, upper respiratory infection, gastritis, conjunctivitis, bilateral leg pain, hip pain, left thigh contusion, influenza, viral syndrome, headache, diarrhea, edema on knee, rib fracture, neuropathy, bilateral varicose veins, gastroenteritis, nasal congestion, chronic cough and anemia. Only one adverse event across all studies was determined by the investigator to be related to treatment.

Each study assessed the following safety variables with no treatment-related adverse findings: clinical laboratory tests (chemistry, hematology, urinalysis), physical exam, vital signs, safety ECG and local skin tolerability.

The Investigator's Brochure includes a complete description of the clinical experience of dapansutrile in all dosage forms. 


\section{TRIAL OBJECTIVES}

The objectives of this clinical trial are as follows:

1. To assess the safety and tolerability of dapansutrile capsules after oral administration in subjects with chronic symptomatic, clinically stable, systolic heart failure (left ventricle ejection fraction $[\mathrm{LVEF}] \leq 40 \%$ and New York Heart Association functional classification II-III [NYHA II-III])

2. To assess the plasma pharmacokinetic concentrations of dapansutrile following oral administration of dapansutrile capsules

3. To assess the pharmacodynamics effect of dapansutrile following oral administration of dapansutrile capsules as measured by changes in inflammatory biomarkers

These objectives will be addressed by the specific outcomes outlined in Section 7 of this protocol.

\section{INVESTIGATIONAL PLAN}

\subsection{OVERALL STUDY DESIGN AND PLAN}

This is a Phase $1 \mathrm{~b}$ randomized, double-blinded, single-center safety and pharmacodynamics study of sequential cohort, dose-escalating, repeat-dosing of dapansutrile or placebo (4:1 ratio) in subjects with stable systolic heart failure (HF) with $\mathrm{LVEF} \leq 40 \%$ symptomatic for NYHA functional classification II-III who show signs of systemic inflammation (plasma $\mathrm{C}$ reactive protein [CRP] or high sensitivity plasma CRP [hsCRP] $>2 \mathrm{mg} / \mathrm{L}$ ). A total of approximately 30 subjects will be randomized in 3 sequential cohorts by randomized allocation ( 8 active and 2 placebo within each cohort). Progression of dose escalation from Cohort 1 to Cohort 2 will occur following the Day 28 visit of the last subject in cohort 1; from Cohort 2 to Cohort 3, progression will occur following the Day 8 visit of the last subject and the Day 28 visit of all other subjects.

Subjects will be screened and evaluated twice for eligibility: 1) at the time of Screening (up to 28 days prior to randomization); and 2) at the Baseline visit, prior to randomization. At Baseline, assessments will be conducted and if the subject is eligible, the first dose of investigational product (either dapansutrile capsules or placebo capsules) will be administered at the clinical site upon completion of all assessments and collection of baseline parameters. Subjects will then self-administer investigational product once, twice or four times daily, depending on cohort, for up to fourteen (14) consecutive days beginning at the Baseline visit and continuing through the planned Day 14 visit. Subjects will return to the study clinic on Days 4 ( \pm 1 day), 8 ( \pm 2 days), 14 ( -4 days) and 28 ( \pm 2 days) for follow-up visits. Investigational product administration will occur under supervision of site personnel on all 
study treatment days involving a site visit. Additionally, it is suggested (not required) that subjects be contacted by telephone for dosing compliance confirmation approximately every 1-2 days during the treatment period. Subjects will also receive a follow-up telephone call on Day 42 ( \pm 3 days).

Safety and tolerability will be evaluated by monitoring the occurrence of adverse events (AEs) and changes in abbreviated physical examination findings, vital signs and clinical safety laboratory test results (chemistry, hematology and cardiac biomarkers), ECGs and body composition measured by bio-impedance analysis. Pharmacodynamics/biomarker endpoints will include blood sampling for plasma hsCRP, IL-1 $\beta$ and interleukin-6 (IL-6), among others. Assessment of cardiorespiratory fitness will include measurement of peak oxygen consumption (peak $\mathrm{VO}_{2}$ ), and ventilatory efficiency $\left(\mathrm{VE} / \mathrm{VCO}_{2}\right.$ slope) using cardiopulmonary exercise testing (CPX). Cardiac systolic and diastolic function will be measured with transthoracic Doppler echocardiography and quality of life questionnaires (Kansas City Cardiomyopathy Questionnaire and Duke Activity Status Index). Additionally, blood samples will be collected for pharmacokinetic assessment of dapansutrile exposure.

\subsection{STUDY DESIGN RATIONALE}

This trial is being performed to assess the safety, pharmacokinetics and pharmacodynamics of dapansutrile capsules when orally administered to subjects with NYHA II-III systolic heart failure for 14 consecutive days. This trial will be conducted in compliance with this protocol, Good Clinical Practices (GCPs) and all applicable regulatory requirements.

This is the first clinical trial of dapansutrile in subjects with heart failure. Based on preclinical toxicology testing, dapansutrile has a clean safety profile to a human equivalent dose of $10 \mathrm{~g} /$ day. A clean human safety profile was additionally demonstrated in the Phase 1 single and multiple dose escalation study of dapansutrile capsules (up to $1 \mathrm{~g}$ /day for 8 days) in normal healthy volunteers. In addition, in the Phase 2 study of subjects with an acute gout flare (Study OLT1177-05) subjects received up to 2,000 mg dapansutrile per day for 8 days. Eight (8) of the 34 enrolled subjects received 2,000 $\mathrm{mg}$ dapansutrile per day (500 $\mathrm{mg}$ QID) with 6 of these $8(75 \%)$ subjects reporting a total of 7 AEs of which 6 were instances of gout or gout flare and were considered mild in severity and possibly related to dapansutrile capsules. The remaining AE was an unrelated, mild severity instance of joint injury after a fall during sport. (These OLT1177-05 results are preliminary and unvalidated as of 05 March 2019). Therefore, the doses selected for study have been qualified through preclinical and/or clinical trials.

Due to its mechanism of action, OLT1177 may be an efficacious IL-1 $\beta$-modulating therapy to treat inflammation in subjects with cardiovascular disease. In support of this hypothesis, OLT1177 was studied in an experimental mouse model of AMI due to ischemia reperfusion injury (Toldo 2017, Toldo 2019). Mice treated with OLT1177 (6, 60 or $600 \mathrm{mg} / \mathrm{kg}$ 
intraperitoneal dose) were shown to have reduced infarct size and improved left ventricle function compared to the vehicle-treated group. Specifically, experimental AMI was induced in adult male mice by ligation of the proximal left coronary artery for 30 minutes followed by release and reperfusion as described previously (Toldo 2011, Abbate 2008).

OLT1177 treatment at reperfusion showed significant dose-dependent reduction in infarct size $(-36 \%,-67 \%$, and $-62 \%$ for 6,60 , and $600 \mathrm{mg} / \mathrm{kg}$, respectively; $\mathrm{P}<0.001$ for linear trend, $\mathrm{P}=0.010$ vs. vehicle for $6 \mathrm{mg} / \mathrm{kg}$, and $\mathrm{P}<0.001$ vs. vehicle for 60 and $600 \mathrm{mg} / \mathrm{kg}$ ). Cardiac systolic function as left ventricular fractional shortening was preserved at 24 hours and 7 days after injury ( $\mathrm{P}=0.015$ for $6 \mathrm{mg} / \mathrm{kg}$ and $\mathrm{P}<0.01$ for 60 and $600 \mathrm{mg} / \mathrm{kg}$ ). OLT1177 reduced infarct size also when given after 60 minutes of reperfusion $(271 \%, \mathrm{P}<0.001$ vs. vehicle). (Toldo 2019).

Results from this study are intended to assess the safety profile of dapansutrile when administered to subjects with systolic heart failure (a common comorbidity in other disease indications for which dapansutrile is being studied) and to enable hypothesis generation for possible applications of dapansutrile in cardiovascular disease indications.

\subsubsection{Selection of Study Population}

\subsubsection{Overview}

One site will randomize a total of approximately 30 subjects at Virginia Commonwealth University hospital in Richmond, VA, USA. The enrollment will be adjusted for those subjects who do not complete the study through the Day 14 visit, so that a total of 30 subjects complete the study.

The study population will be comprised of English-speaking male and female subjects 18 years or older with stable systolic heart failure (HF) with $\mathrm{LVEF} \leq 40 \%$ symptomatic for NYHA functional classification II-III who show signs of systemic inflammation (plasma $\mathrm{C}$ reactive protein [CRP] or high sensitivity plasma CRP [hsCRP] $>2 \mathrm{mg} / \mathrm{L}$ ). This population was selected as the investigational product, dapansutrile, is being developed for multiple therapeutic applications in which heart failure is a common comorbidity. Additionally, given the established activity of anti-IL-1 therapies and the preliminary data showing a cardioprotective effect of dapansutrile, studying this population will provide a foundation for studying subject populations suffering from a variety of cardiovascular disease indications in future studies.

While heart failure is not specific to the geriatric population, the risk of heart failure increases with age and the target population is likely to include geriatric subjects. As such, the potential inclusion of geriatric subjects in this study is warranted to discern early indications of any differences present in this population. 


\subsubsection{Recruitment}

Recruitment of study participants will be conducted by the Investigator and/or site staff and may utilize the following methods:

- Request for referral from general practitioners

- Direct recruitment of participants, including subjects of the Investigator and/or the study clinic

- Local advertisement with IRB-approved recruitment materials (e.g., flyers, posters, radio advertisement, television advertisement, etc.)

- Word of mouth

\subsubsection{Inclusion Criteria}

A subject may be included in the study if he/she meets each of the criteria as presented below:

1) Male and female subjects 18 years old or older

2) Symptomatic stable HF (NYHA class II-III) with reduced left ventricular ejection fraction (LVEF $\leq 40 \%$, measured within 6 months of enrollment - no changes in cardiac medications or new device implantation within past 2 months)

3) Peak exercise limited by shortness of breath and/or fatigue associated with a respiratory exchange ratio $($ RER) $>1.00$ (reflecting maximal aerobic effort)

4) Reduced peak aerobic exercise capacity (peak $\mathrm{VO}_{2}$ ) to less than $80 \%$ of predicted value by age/gender at Baseline

5) Plasma CRP or hsCRP levels $>2 \mathrm{mg} / \mathrm{L}$ at Screening

6) Acceptable overall medical condition to be safely enrolled in and to complete the study (with specific regard to cardiovascular, renal and hepatic conditions) in the opinion of the Principal Investigator

7) Ability to provide written, informed consent prior to initiation of any study-related procedures, and ability, in the opinion of the Principal Investigator, to understand and comply with all the requirements of the study

\subsubsection{Exclusion Criteria}

A subject may not be included if he/she meets one or more of the criteria presented below:

1) Women of childbearing potential, or men whose sexual partner(s) is a woman of childbearing potential who:

a. Are or intend to become pregnant (including use of fertility drugs) during the study 
b. Are nursing

c. Are not using an acceptable, highly effective method of contraception until all follow-up procedures are complete. (Acceptable, highly effective forms of contraception are defined as: oral contraception, intrauterine device, systemic [injectable or patch] contraception, double barrier methods, naturally or surgically sterile, strict abstinence or partner has been sterilized. If hormonalbased birth control is being used, subject or subject's sexual partner(s) must be on a stable-dose for $\geq 3$ months prior to the Baseline visit and maintained at the same dosing level throughout the study.)

2) Abnormal blood pressure or heart rate response, angina or ECG changes (ischemia or arrhythmias) occurring during CPX

3) Presence or known history of autoimmune conditions (e.g., systemic lupus erythematosus, hypophysitis, etc.)

4) History or evidence of active tuberculosis (TB) infection at Baseline visit or one of the risk factors for tuberculosis such as but not limited or exclusive to:

a. History of any of the following: residence in a congregate setting (e.g., jail or prison, homeless shelter, or chronic care facility), substance abuse (e.g., injection or non-injection), health-care workers with unprotected exposure to subjects who are at high risk of $\mathrm{TB}$ or subjects with $\mathrm{TB}$ disease before the identification and correct airborne precautions of the subject

or

b. Close contact (i.e., share the same air space in a household or other enclosed environment for a prolonged period (days or weeks, not minutes or hours)) with a person with active pulmonary TB disease within the last 12 months.

5) Use of any prohibited concomitant medications/therapies over the periods defined in Section 5.6.3 or planned use of any prohibited concomitant medications/therapies during the Treatment Period

6) Any other concomitant medical or psychiatric condition(s), disease(s) or prior surgery(ies) that, in the opinion of the Principal Investigator, would impair the subject from safely participating in the trial and/or completing protocol requirements, including but not limited to:

a. physical inability to walk on a treadmill

b. decompensated HF (edema, NYHA IV)

c. significant ischemic heart disease, angina

d. arterial hypotension (blood pressure [BP] systolic $<90 \mathrm{mmHg}$ )

e. arterial hypertension (resting BP systolic $>160 \mathrm{mmHg}$ ) 
f. atrial fibrillation with rapid ventricular response

g. severe valvular disease

h. severe chronic obstructive or restrictive pulmonary disease

i. moderate-severe anemia $(\mathrm{Hgb}<10 \mathrm{~g} / \mathrm{dL})$

j. severe diabetic neuropathy or myopathy

7) Active or recent (within 2 weeks) infection prior to the Baseline visit

8) History of or known positive for HIV, Hepatitis B surface antigen or antibodies to Hepatitis C Virus

9) Known history of renal impairment and/or creatinine clearance less than $50 \mathrm{~mL} / \mathrm{min}$ calculated by Cockcroft-Gault method

10) Active malignancy or recent malignancy with chemotherapy treatment within the past 6 months

11) Enrollment in any trial and/or use of any investigational product or device within the immediate 30-day period prior to the Baseline visit

12) Previous exposure to the investigational product

\subsubsection{Early Termination of Subjects from Therapy}

Subjects will be informed, prior to the trial's commencement, that they will have the right to withdraw from the trial at any time, for any reason, without prejudice to their safety or medical care. Moreover, Investigators will have the right to withdraw a subject from the trial if there is any reason, in the Investigator's opinion, that would impair the subject from safely participating in the trial, including completing any protocol requirements.

Notification of early termination due to adverse event or any other safety-related concerns will immediately be made to the Medical Monitor and Sponsor or Sponsor's Representative. The date the subject is withdrawn from the trial and the reason for discontinuation will be recorded in the source documents and in the subject's CRF. If a subject withdraws from the trial because of an adverse event, the principal specific event and any related test results will be recorded in the CRF in both the End of Study and the Adverse Event CRF pages. Follow-up of any persisting adverse event is required until the event resolves or stabilizes at a level acceptable to the Investigator or Investigator's designee.

Reasons for early termination may include, but are not limited to:

- Adverse Events (AEs)

- Worsening condition, as determined by the Investigator 
- Withdrawal of consent

- Changes in the subject's condition that would impair the subject from safely participating in the trial, including but not limited to completing any protocol requirements, in the judgment of the Investigator

- Subject becomes pregnant (withdrawal is required)

- $\quad$ Subject is lost to follow-up

\subsubsection{Subject-Specific Stopping Rules}

If an enrolled subject experiences any of the following, investigational product treatment will be immediately ceased and the subject will be discontinued from the study:

- Serious adverse event that is deemed to be possibly related, probably related or related to the investigational product;

- Change in systolic blood pressure, diastolic blood pressure or heart rate that exceeds $25 \%$ from baseline that is, in the opinion of the Principal Investigator, not due to another identifiable cause (e.g., non-compliance with other medications);

- New or worsening chest pain that is considered to be likely or possibly related to myocardial ischemia (angina) or elevated filling pressures (heart failure);

- New or worsening ischemic ECG changes, defined as any of the following: new or worsening ST segment deviation $>0.5 \mathrm{~mm}$ and/or T wave inversion in 2 anatomically contiguous leads;

- Abnormal cardiac rhythm including: any symptomatic ventricular or supraventricular arrhythmia; asymptomatic ventricular arrhythmia that is sustained more than 30 seconds or requires treatment; asymptomatic atrial fibrillation that is sustained more than 60 seconds; asymptomatic supraventricular tachycardia that is sustained more than 10 minutes or requires treatment; a systolic pause $>3$ seconds; a second degree type II or third degree AV block in a patient without pacemaker;

- Change in body weight that exceeds $10 \%$ from Baseline that is, in the opinion of the Principal Investigator, not due to another identifiable cause (e.g., non-compliance with other medications);

- NYHA class IV heart failure symptoms;

- Acute infection (of any kind);

- Non-compliance with the protocol by the subject in a way that the Principal investigator is concerned about the safety of the subject and/or the accuracy of the data;

- Any new incident condition that may impair the subject to safety complete the study and/or for the Principal Investigator to accurately gather the data. 


\subsubsection{Early Discontinuation of the Trial}

The Sponsor and the Medical Monitor reserve the right to discontinue the trial in accordance with applicable law. Reasons for discontinuation may include, but are not limited to:

- Approval by the IRB is irrevocably revoked

- In interest of the health of the trial subjects

- Determination that continuation of the trial cannot serve a scientific purpose

- Unremedied violation of applicable law, GCPs or this study protocol by the Investigator

- A request to discontinue the trial from a regulatory authority

- Sponsor business decision

\subsubsection{Study-Level Stopping Rules}

If any of the following occur, the study will be temporarily suspended and will not be restarted until a meeting is held between the Medical Monitor, Principal Investigator, Sponsor and an independent cardiologist to determine the appropriate course of action:

- Any serious adverse event that is possibly, probably or related to investigational product;

- After the first 3 or more subjects are enrolled, if: (1) $50 \%$ or more subjects experience a serious adverse event considered unrelated to the investigational product; or (2) $75 \%$ or more subjects experience either a serious adverse event unrelated to the investigational product or a non-serious adverse event of Grade 2 or 3 intensity that is possibly, probably or related to the investigational product.

Procedurally, the Medical Monitor or designee will schedule and convene a meeting by teleconference. Attendance by the Medical Monitor, Principal Investigator, a Sponsor representative and an independent cardiologist is required in order for a meeting to be held. A meeting agenda and any pertinent study data (e.g. tables, listings) will be distributed prior to the meeting, formal meeting minutes will be kept including documentation of any resolutions or recommendations reached during the meeting. A unanimous decision is required if the recommendation conclusion during this meeting is to resume enrollment in the study. The meeting materials - which include the meeting agenda, data package and meeting minutes will be submitted to the IRB at which time the study may resume, if such a determination is unanimously reached. 


\section{TREATMENTS}

\subsection{TREATMENTS ADMINISTERED}

Dapansutrile capsules (100 mg dapansutrile each) or placebo capsules will be self-administered for the duration of the Treatment Period beginning at the Baseline visit and will continue through the planned Day 14 visit. Investigational product will be self-administered orally once (Cohort 1), two times (Cohort 2) or four times (Cohort 3) per day. Investigational product administration will occur under supervision of site personnel on all study treatment days involving a site visit.

\subsection{IDENTITY OF INVESTIGATIONAL PRODUCT}

\subsubsection{Description of Investigational Product}

Subjects will be assigned to one of two investigational product types: dapansutrile capsules or identical Placebo Capsules. Dapansutrile capsules consist of a blend of approximately $100 \mathrm{mg}$ dapansutrile API with Avicel PH-101. Placebo Capsules consist of only Avicel PH-101. Both dapansutrile capsules and Placebo Capsules are encapsulated in a hard, opaque white capsule and are identical by physical inspection.

\subsubsection{Packaging of Investigational Product}

Investigational product will be packaged in 20-count HDPE round bottles. Each bottle will be labeled to display (at a minimum) its Bottle Number and will contain space for the assigned Subject Identification Number to be written by site personnel.

\subsection{DISPENSING OF INVESTIGATIONAL PRODUCT}

Investigational product will be dispensed to subjects at the following visits: Baseline, Day 4 and Day 8. Subjects will return all dispensed bottles of investigational product at each subsequent visit and will be given a sufficient quantity of investigational product to enable dosing until the next dispensation.

\subsection{METHOD OF ASSIGNING SUBJECTS TO TREATMENT GROUPS}

\subsubsection{Randomization}

Subjects will be randomized in a ratio of 8 active to 2 placebo within each cohort. The Sponsor's independent statistician will generate the randomization scheme, which will then be provided to the Sponsor's designated unblinded clinical trial supply management vendor. Upon site activation, the Sponsor's clinical trial supply management vendor will distribute a randomized set of investigational product bottles to the clinical site. The site will in turn 
randomize subjects who meet all eligibility criteria and will sequentially allocate a labeled bottle(s) of investigational product to the subject.

\subsubsection{Subject Identification (SID) Number}

All screened subjects will be assigned a six-digit subject identification (SID) number, of which the first three digits will be the clinical site identification number and the last three digits will be the subject number. The SID numbers at the clinical site will be assigned sequentially in the order subjects are screened. The SID number identifies the subject from the time of screening through the duration of the trial. For example, the fifth subject screened at the site assigned clinical site identification number 001 would be assigned SID: 001-005.

\subsubsection{Cohort Progression}

Approximately ten (10) subjects will be randomized in the first cohort with replacement allowed for subjects who discontinue before Day 14. Upon completion of the Day 28 visit by all subjects in Cohort 1, an interim data analysis will be conducted and reviewed by the Medical Monitor, an independent cardiologist and the Principal Investigator and a decision about progression of the study to enroll Cohort 2 will be made.

In the second cohort, approximately ten (10) subjects will be randomized with replacement allowed for subjects who discontinue before Day 14. Upon completion of the Day 8 visit by the last subject in Cohort 2 and of the Day 28 visit by all other subjects, an interim data analysis will be conducted and reviewed by the Medical Monitor, an independent cardiologist and the Principal Investigator and a decision about progression of the study to enroll Cohort 3 will be made.

\subsection{BLINDING OF THE STUDY}

Every effort should be made to maintain the integrity of the blind. Only the Sponsor's independent statistician and the Sponsor's clinical trial supply management vendor will have access to the randomization scheme / drug assignment list. In addition, the Sponsor's Medical Monitor for the study will be unblinded to treatment assignment in order to monitor the safety of subjects. All other parties will remain blinded to all subjects' treatment assignment until completion of the Day 42 follow-up call of the full cohort, including but not limited to: the Sponsor, the Sponsor's Representatives, subjects and trial personnel, such as Investigators, trial coordinators, nursing staff and study monitors.

Each kit will have a detachable label with the randomization number. The label will be covered by an opaque, scratch-off film, which will conceal the treatment group information. The Investigator will have the authority to scratch off the film for a particular subject in the event of a medical emergency or a serious adverse event necessitating the identification of the test material for the welfare of the subject. The Investigator will document in the CRF, the reason 
for removing the blind, as well as the date and time, all of which will be affirmed by his/her signature. The Investigator will be instructed to call the Medical Monitor and/or the Sponsor Representative prior to breaking the blind, unless the subject's safety requires breaking the blind prior to notification.

Upon completion of the Day 28 visit by all subjects in Cohort 1, the treatment assignments for the completed cohort may be unblinded to the study-designated bioanalytical / pharmacokinetics lab. Upon completion of the Day 8 visit by the last subject in Cohort 2 and the Day 28 visit by all other subjects in Cohort 2, the treatment assignments for the completed cohort may be unblinded to the study-designated bioanalytical / pharmacokinetics lab.

\subsection{PRIOR AND CONCOMITANT MEDICATIONS/THERAPIES}

\subsubsection{Allowed Concomitant Medications/Therapies}

Any medical conditions during the Treatment Period of the trial will be treated at the discretion of the Investigator according to acceptable community standards of medical care. All concomitant medications and therapies will be documented in the subject's CRF.

Subjects will be allowed to continue taking stable doses of concomitant medication, except those medications/therapies listed under "Prohibited Concomitant Medications/Therapies" in Table 5.1.

\subsubsection{Contraception Protection}

All women of childbearing potential enrolling in the trial must agree to use an acceptable, highly effective method of contraception. Men enrolling in the trial and whose sexual partner(s) is/are a woman of childbearing potential, must also agree to use an acceptable, highly effective method of contraception. Acceptable, highly effective forms of contraception are defined as: oral contraception, intrauterine device, systemic (injectable or patch) contraception, double barrier methods, naturally or surgically sterile, strict abstinence or partner has been sterilized. If hormonal-based birth control is being used, subject or subject's sexual partner(s) must be on a stable-dose for $\geq 3$ months prior to the Baseline visit and maintained at the same dosing level throughout the study.

\subsubsection{Prohibited Concomitant Medications/Therapies}

The following concomitant medications/therapies are prohibited except under the specific conditions noted. Subjects taking these medications should either be excluded from the trial as noted in the entry criteria, or if on a permitted stable dose, should continue on their stable-dose treatment for the full 14-day Treatment Period (under specific conditions noted). 
Subjects who enroll in this trial should not begin use of, or intermittently use, any prohibited concomitant medication/therapy during the 14-day Treatment Period. (PRN dosing is not considered a stable dose.)

Table 5.1 summarizes those medications/therapies that are prohibited (except as otherwise noted).

Table 5.1 Prohibited Concomitant Medications/Therapies

\begin{tabular}{|c|c|c|}
\hline $\begin{array}{l}\text { Medication / Therapy } \\
\text { Class }\end{array}$ & $\begin{array}{c}\text { Conditions for } \\
\text { Enrollment/Allowance }\end{array}$ & Examples \\
\hline $\begin{array}{l}\text { Systemic } \\
\text { corticosteroids }\end{array}$ & $\begin{array}{l}\text { Last dose taken must have been at least } \\
2 \text { weeks prior to Baseline visit }\end{array}$ & $\begin{array}{l}\text { Prednisone, cortisone, } \\
\text { methylprednisolone, etc. }\end{array}$ \\
\hline $\begin{array}{l}\text { Other investigational } \\
\text { products }\end{array}$ & $\begin{array}{l}\text { Last dose taken must have been at least } \\
30 \text { days prior to Baseline visit }\end{array}$ & Any drug not approved by FDA \\
\hline $\begin{array}{l}\text { Cancer } \\
\text { chemotherapeutic } \\
\text { drugs }\end{array}$ & Not allowed in the past 6 months & $\begin{array}{l}\text { Carboplatin, cisplatin, } \\
\text { cyclophosphamide, doxorubicin, } \\
\text { etoposide, fluorouracil, gemcitabine, } \\
\text { irinotecan, methotrexate, paclitaxel, } \\
\text { topotecan, vincristine, vinblastine, etc. }\end{array}$ \\
\hline Immunotherapies & $\begin{array}{l}\text { Last dose taken must have been at least } \\
5 \text { half-lives prior to the Baseline visit }\end{array}$ & $\begin{array}{l}\text { Interferons, interleukins, anti-TNF } \\
\text { therapy, monoclonal antibodies, colony } \\
\text { stimulating factors (CSFs) }\end{array}$ \\
\hline Live vaccinations & $\begin{array}{l}\text { Last live vaccination should be at least } \\
2 \text { weeks prior to the Baseline visit }\end{array}$ & $\begin{array}{c}\text { Measles, mumps, and rubella } \\
\text { (MMR) vaccine, varicella vaccine, etc. }\end{array}$ \\
\hline
\end{tabular}

Subject safety must always remain paramount. Therefore, if an Investigator deems that a subject requires a prohibited concomitant medication/therapy, at the Investigator's discretion and according to acceptable community standards of medical care, the subject will receive concomitant medication/therapy as specified. The subject may be withdrawn from the study at the discretion of the Investigator, in consultation with the Sponsor and Medical Monitor for use of concomitant medications/therapies that are prohibited.

\subsection{TREATMENT COMPLIANCE}

During the 14-day Treatment Period, subjects will report the administration of investigational product either once (Cohort 1), twice (Cohort 2) or four times (Cohort 3) daily using the supplied dosing diary. The site personnel will have access to each subject's diary at the site visits and will track subject compliance with the investigational product on an ongoing basis. It is suggested (not required) that Site staff contact subjects by phone approximately every 12 days to confirm dosing compliance. 


\subsection{INVESTIGATIONAL PRODUCT STORAGE \& ACCOUNTABILITY}

\subsubsection{Storage}

Investigational product should be stored in a secure area that is maintained at room temperature: $15^{\circ} \mathrm{C}-25^{\circ} \mathrm{C}\left(59^{\circ} \mathrm{F}-77^{\circ} \mathrm{F}\right)$. Slight deviations from this temperature range are acceptable. Significant deviations should be recorded and the Sponsor should be contacted to determine any effect on the investigational product.

\subsubsection{Accountability}

Upon receipt of the investigational product, the Investigator is responsible for ensuring that the designated site personnel conduct a complete inventory of trial materials and the Investigator assumes responsibility for their storage and dispensing. In accordance with applicable regulations, the Investigator must agree to keep all trial materials in a secure location with restricted access. The Investigator will keep a record of the inventory and dispensing of all investigational product. This record will be made available to the study monitor or other qualified representatives of the Sponsor for the purpose of accounting for all clinical supplies. Any significant discrepancy and/or deficiency must be recorded with an explanation.

All supplies sent to the Investigator will be accounted for and, in no case, used in any unauthorized situation.

Used (empty), unused or expired bottles of the investigational product are to be collected and saved at the site for inspection by the study monitor of other qualified representatives of the Sponsor throughout the study. 


\section{SAFETY, PHARMACODYNAMICS AND DISEASE-STATE VARIABLES}

\subsection{SAFETY VARIABLES}

Safety variables collected in the study will be:

- Physical examination (abbreviated general and site specific examination)

- Vital Signs (pulse, resting blood pressure, temperature, respiration rate)

- Safety laboratory measures (chemistry, hematology and cardiac biomarkers [plasma NT-proBNP levels])

- Safety electrocardiograms (ECGs)

- Bio-impedance analysis

- Cardiopulmonary exercise test

- Transthoracic Doppler echocardiography

- Adverse events during the clinical trial

\subsection{PHARMACODYNAMICS / BIOMARKER VARIABLES}

Blood samples will be collected for PD/biomarker analysis, including:

- High-sensitivity C-reactive protein (hsCRP) [analyzed as part of safety labs: chemistry]

- Levels of circulating cytokines (e.g., IL-1ß, IL-6)

\subsection{PHARMACOKINETIC VARIABLES}

Pharmacokinetic criteria for evaluation are as follows:

- Plasma concentrations of dapansutrile

\subsection{QUALITY OF LIFE AND PAIN VARIABLES}

Subjects will complete the following procedures, designed to assess quality of life or pain metrics:

- Kansas City Cardiomyopathy Questionnaire

- Duke Activity Status Index

- Brief Pain Index 


\section{TRIAL ASSESSMENTS}

Trial assessments will be completed at each visit to the study clinic. Additionally, subjects will be supplied with a study diary to record: dosing compliance, adverse events and concomitant medication use.

Available medical data collected/performed within 72 hours prior to the Screening or Baseline visits may be used to satisfy the protocol-specified study procedures.

At each of the Baseline, Day 4 ( \pm 1 day), Day 8 ( \pm 2 days), and Day 14 (- 4 days) visits, investigational product will be administered under supervision of site personnel.

\subsection{SAFETY}

\subsubsection{Medical History and Concomitant Medications/Therapies Review}

A review of each subject's pertinent medical history, including medication history, within the past 5 years, taking into account all recent pertinent medical conditions, will be recorded at the Screening visit and reviewed and updated at the Baseline visit. Studies will be dispensed a Study Diary which will include a section to record use of concomitant medications/therapies.

All concomitant medications/therapies and past medications/therapies will be reviewed and recorded in the CRF at the following visits: Screening, Baseline (prior to dosing; including those taken within the past 30 days), Day 4 ( \pm 1 day), Day 8 ( \pm 2 days), Day 14 ( -4 days $)$ and Day 28 ( \pm 2 days).

\subsubsection{Physical Examination}

A full physical examination will be performed at the Screening visit. An abbreviated / targeted physical examination will be performed at the Baseline, Day 14 (- 4 days) and Day 28 ( \pm 2 days) visits (or at an early termination visit if before the Day 28 visit) based on the subject's reported symptoms. Additional targeted physical examinations may be conducted at the discretion of the Investigator, if required by the subject's signs and symptoms. Height will be recorded as part of the physical examination at the Screening visit only. Weight will be collected at each study visit regardless of the performance of a full or abbreviated/targeted physical examination.

\subsubsection{Vital Signs}

Vital signs (pulse, resting blood pressure [must be resting for 10 minutes], temperature and respirations) will be recorded at the following visits: Screening, Baseline (prior to dosing), Baseline (post-dose), Day 4 ( \pm 1 day), Day 8 ( \pm 2 days), Day 14 (- 4 days) and Day 28 ( \pm 2 days) or early termination (if before the Day 28 visit). For Baseline (post-dose) and Days 4, 8 
and 14, vital signs will be recorded at 1 hour ( $\pm 15 \mathrm{~min})$ after investigational product administration.

\subsubsection{Urine Pregnancy Test}

Women who have undergone hysterectomy, bilateral oophorectomy, bilateral tubal ligation or have been without menses for 12 months are considered to be of non-child bearing potential. All other women will require a urine pregnancy test during each of the following visits: Screening, Baseline (prior to dosing) and Day 28 ( \pm 2 days) or at an early termination visit (if before Day 28 visit).

\subsubsection{Safety Laboratory Measures}

Blood will be drawn for safety laboratory measures (hematology, chemistry and cardiac biomarkers) at the following visits: Screening, Baseline (Day 1; pre-dose), Day 4 ( \pm 1 day), Day 8 ( \pm 2 days), Day 14 (- 4 days) and Day 28 ( \pm 2 days) or at an early termination visit (if before the Day 28 visit). For Days 4, 8 and 14, draws will occur at 1 hour ( $\pm 15 \mathrm{~min}$ ) after investigational product administration. In addition to these safety laboratory measures, hsCRP (a PD biomarker analyzed as part of safety labs: chemistry) will also be drawn during Baseline visit after the first dose has been administered (Baseline / Day 1; post-dose).

Confirmatory or follow-up laboratory measurements may be taken at the discretion of the Investigator as warranted by the subject's signs and symptoms (e.g., clinically significant abnormal laboratory values at previous visit).

\subsubsection{Hematology}

The following hematology testing should be performed at each time point: CBC with 5-part differential, RBC statistics and platelet count.

\subsubsection{Chemistry}

The following chemistry testing should be performed at each time point: total bilirubin, alanine transaminase (ALT), aspartate transaminase (AST), total protein, albumin, lipase, sodium, potassium, calcium, blood urea nitrogen (BUN), creatinine, cystatin $\mathrm{C}$, glucose, chloride, glycated haemoglobin (HbA1c), high sensitivity C-reactive protein (hsCRP). Additionally, $\mathrm{C}$-reactive protein (CRP) may be tested at the Screening and/or Baseline visit at the discretion of the Investigator.

\subsubsection{Cardiac Biomarkers}

The following cardiac biomarker testing should be performed at each time point: plasma NT-proBNP. 


\subsubsection{Safety ECGs}

12-lead ECGs will be collected at the following visits: Baseline (Day 1; pre-dose and postdose), Day 4 ( \pm 1 day) and Day 8 ( \pm 2 days). For Baseline (post-dose) and Days 4 and 8, ECG collection will occur at 1 hour $( \pm 15 \mathrm{~min})$ after investigational product administration. Subjects must be resting for at least 10 minutes prior to obtaining ECGs. Additional ECGs will be collected as part of the Cardiopulmonary Exercise procedure (see Section 7.1.8).

\subsubsection{Bio-impedance Analysis}

Bio-impedance or Bioelectrical Impedance Analysis (BIA) is a non-invasive, quick and safe technique to estimate body composition compartments (total body water, intracellular water, extracellular water, fat mass, fat-free mass, lean mass). Impedance is defined as the property of the electrical ionic conduction of soft tissue where fat and bone are considered poor conductors; the microampere range developed during the test does not represent any hazard for the subject. The subject will be asked to stay still on the bed, with superior limbs abducted at approximately $30^{\circ}$ and the inferior ones at approximately $45^{\circ}$. Four cutaneous electrodes (two on the foot and two on the homolateral hand) will be applied with at least $5 \mathrm{~cm}$ distance between electrodes. A small electric current is applied to the electrodes. Electric conductivity will be measured through the body using a single-frequency Quantum IV Body Composition Analyzer RJL Systems and then, using a dedicated software (RJL Systems), we will determine body composition compartments. The estimated duration of the study is less than $10 \mathrm{~min}$.

This assessment will be completed at the Screening, Baseline (Day 1; pre-dose), Day 14 (- 4 days) and Day 28 ( \pm 2 days) visits. However, this assessment is considered optional during the Screening visit. If BIA is completed during the Screening visit and the Screening visit and Baseline (Day 1; pre-dose) visit occur within 72 hours, then BIA does not need to be repeated at the Baseline (Day 1; pre-dose) visit.

\subsubsection{Cardiopulmonary Exercise Test (CPX)}

Subjects will be scheduled for a cardiopulmonary exercise test (CPX). American College of Cardiology/American Heart Association guidelines for exercise testing contraindications and test termination criteria will be followed (Fletcher 2013). A physician-supervised maximal aerobic exercise test will be administered using a metabolic cart that is interfaced with a motorized treadmill. A conservative ramping protocol will be used with incremental increases in workload of approximately 0.6 metabolic equivalents per minute. Prior to each test, the oxygen and carbon dioxide sensors will be calibrated using gases of known oxygen, nitrogen, and carbon dioxide concentrations and the pneumotachometer will undergo volume-calibration using a 3-liter syringe. Subjects will be briefed regarding the protocol and will be requested to exercise to volitional fatigue. 12-lead ECG monitoring will be conducted at baseline, throughout the test and into recovery. Blood pressure will be measured every two minutes 
using an automated exercise-compatible device (Tango, SunTech Medical). During CPX, expired gases are sampled and analyzed to continuously measure oxygen uptake $\left(\mathrm{VO}_{2}\right)$ and carbon dioxide production $\left(\mathrm{VCO}_{2}\right)$; the highest 10 -second interval average value over the last 30 -seconds of exercise will be used to define peak oxygen consumption (peak $\mathrm{VO}_{2}$ ). Percent predicted $\mathrm{VO}_{2}$ calculations will be based upon the equations of Wasserman et al., (1999). The respiratory exchange ratio (RER) will be determined by the $\mathrm{VCO}_{2} / \mathrm{VO}_{2}$ ratio that corresponds to the peak $\mathrm{VO}_{2}$. The ventilatory equivalents method will be used to determine $\mathrm{VO}_{2}$ at the ventilatory anaerobic threshold. Ten second averaged minute ventilation (VE) and $\mathrm{VCO}_{2}$ data, from the initiation of exercise to peak, will be put into spreadsheet software (Microsoft Excel, Microsoft Corp., Bellevue, WA) to calculate the $\mathrm{VE} / \mathrm{VCO}_{2}$ slope via least squares linear regression $(\mathrm{y}=\mathrm{mx}+\mathrm{b}, \mathrm{m}=$ slope $)$. All CPXs will be performed by an American College of Sports Medicine registered clinical exercise physiologist.

Upon completion of exercise testing, the results of the cardiopulmonary exercise test will be reviewed and discussed with the referring physician(s).

A peak $\mathrm{VO}_{2}<80 \%$ of predicted with evidence of sufficient effort (defined as RER $>1.0$ ) will be considered diagnostic for exercise intolerance.

This assessment will be completed at the Screening, Baseline (Day 1; pre-dose), Day 14 (- 4 days) and Day 28 ( \pm 2 days) visits. However, this assessment is considered optional during the Screening visit. If CPX is completed during the Screening visit and the Screening visit and Baseline (Day 1; pre-dose) visit occur within 72 hours, then CPX does not need to be repeated at the Baseline (Day 1; pre-dose) visit.

\subsubsection{Transthoracic Doppler Echocardiography}

All subjects will undergo a resting transthoracic echocardiogram (TTE) prior to CPX to measure left and right ventricular and atrial dimensions, left and right ventricular systolic function, transmitral flow Doppler spectra, mitral and tricuspid valve annulus tissue Doppler spectra, ejection time and stroke volume, inferior vena cava, aorta and pulmonary artery diameters and Doppler spectra, according to the recommendations of the American Society of Echocardiography. A qualified, trained professional will perform all echocardiography. The results will be interpreted by a cardiologist, and reviewed and discussed with the subject and with the referring physician(s).

This assessment will be completed at the Screening, Baseline (Day 1; pre-dose), Day 14 (- 4 days) and Day 28 ( \pm 2 days) visits. However, this assessment is considered optional during the Screening visit if a previous qualifying TTE ( $\mathrm{LVEF}<40 \%$ ) has been completed during the past 6 months. If TTE is completed during the Screening visit and the Screening visit and Baseline (Day 1; pre-dose) visit occur within 72 hours, then TTE does not need to be repeated at the Baseline (Day 1; pre-dose) visit. 


\subsubsection{Adverse Events}

Adverse events will be collected starting immediately after the subject signs the ICF and continuing through Day 42 ( \pm 3 day). All reported AEs will be followed until resolution or stabilization or until the subject is lost to follow-up. Subjects will be dispensed a Study Diary which will include a section to record AEs.

\subsection{PHARMACOKINETIC \& PHARMACODYNAMICS ASSESSMENTS}

\subsubsection{Pharmacokinetics}

Blood will be drawn for plasma PK analysis at the following time points: Baseline visit (Day 1; pre-dose and post-dose), Day 4 ( \pm 1 day), Day 8 ( \pm 2 days), Day 14 ( 4 days) and Day 28 ( \pm 2 days) visits. For Baseline (post-dose) and Days 4, 8 and 14, draws will occur at 1 hour ( $\pm 15 \mathrm{~min}$ ) after investigational product administration.

Blood samples for plasma PK analysis will be collected into $4 \mathrm{~mL} \mathrm{~K}_{2}$-EDTA (lavender top) anticoagulation tubes at each time point. Samples should be centrifuged and plasma collected, equally divided into two separate aliquots, and placed in $2 \mathrm{~mL}$ cryovials. Shipping materials will be provided for frozen shipment to the Sponsor's designated bioanalysis laboratory. Detailed instructions on handling PK samples will be provided in the PK \& PD Collection Manual.

\subsubsection{Pharmacodynamics}

Blood will be drawn for PD biomarker analysis at the Baseline (Day 1; pre-dose and postdose), Day 4 ( \pm 1 day), Day 8 ( \pm 2 days), Day 14 ( -4 days) and Day 28 ( \pm 2 days) visits. For Baseline (Day 1; post-dose) and Days 4, 8 and 14, draws will occur at 1 hour ( \pm 15 min) after investigational product administration.

PD biomarkers to be assessed include but are not limited to:

- High sensitivity C-reactive protein (hsCRP) [analyzed as part of safety labs: chemistry at all study visits. (See Section 7.1.5)

○ Plasma cytokines, including: IL-1 $\beta$, IL-1Ra, IL-18, IL-6, TNF- $\alpha$

Blood samples for cytokine analysis will be collected into $4 \mathrm{~mL} \mathrm{~K}_{2}$-EDTA (lavender top) anticoagulation tubes at each time point. A full description of the procedures for collection and transfer of cytokine samples is contained in the PK \& PD Sample Collection Manual. Samples will be retained until completion of study analyses which is anticipated to last (but may exceed) a period of approximately 5 years. 


\subsection{QUALITY OF LIFE AND PAIN ASSESSMENTS}

\subsubsection{Kansas City Cardiomyopathy Questionnaire (KCCQ)}

The Kansas City Cardiomyopathy Questionnaire is a 23-item, self-administered instrument that quantifies physical function, symptoms (frequency, severity and recent change), social function, self-efficacy and knowledge, and quality of life. An overall summary score can be derived from the physical function, symptom (frequency and severity), social function and quality of life domains. For each domain, the validity, reproducibility, responsiveness and interpretability have been independently established. Scores are transformed to a range of $0-100$, in which higher scores reflect better health status.

This assessment will be completed at the Screening, Baseline (Day 1; pre-dose), Day 14 (- 4 days) and Day 28 ( \pm 2 days) visits. However, this assessment is considered optional during the Screening visit. If KCCQ is completed during the Screening visit and the Screening visit and Baseline (Day 1; pre-dose) visit occur within 72 hours, then KCCQ does not need to be repeated at the Baseline (Day 1; pre-dose) visit.

\subsubsection{Duke Activity Status Index (DASI)}

The Duke Activity Status Index is a self-administered questionnaire that measures a subject's functional capacity. It is commonly used to obtain a rough estimate of a subject's peak oxygen uptake. This index consists of 12 weighted "yes or no" questions, which are summed and used to calculate VO2peak and METS according to the following equations:

Duke Activity Status Index (DASI) score = sum of "Yes" replies

$\mathrm{VO}_{2}$ peak $=(0.43 \times \mathrm{DASI})+9.6$

METS $=\mathrm{VO}_{2}$ peak $\div 3.5 \mathrm{ml} / \mathrm{kg} / \mathrm{min}$

This assessment will be completed at the Screening, Baseline (pre-dose), Day 14 (- 4 days) and Day 28 ( \pm 2 days) visits. However, this assessment is considered optional during the Screening visit. If DASI is completed during the Screening visit and the Screening visit and Baseline (Day 1; pre-dose) visit occur within 72 hours, then DASI does not need to be repeated at the Baseline (Day 1; pre-dose) visit.

\subsubsection{Brief Pain Index (BPI)}

The Brief Pain Index is a self-administered questionnaire that assesses the severity of pain and the impact of pain on daily functions. It is being used in this study to capture any changes to a subject's existing aches/pains over the duration of the study. 
This assessment will be completed at the Baseline (pre-dose), Day 4 ( \pm 1 day), Day 8 ( \pm 2 days), Day 14 (- 4 days) and Day 28 ( \pm 2 days) visits. 
Table 7.1 Schedule of Trial Activities \& Assessments

\begin{tabular}{|c|c|c|c|c|c|c|c|c|c|}
\hline \multicolumn{2}{|c|}{ Trial Period: } & \multicolumn{2}{|c|}{ Eligibility } & \multicolumn{4}{|c|}{ Treatment } & \multicolumn{2}{|c|}{ Follow-up } \\
\hline \multirow{2}{*}{ Assessment } & \multirow{2}{*}{ Visit: } & \multirow{2}{*}{$\begin{array}{c}\text { Screening } \\
(\text { Day }-28 \text { to } 1)^{\mathrm{h}}\end{array}$} & \multicolumn{2}{|c|}{ Baseline (Day 1) ${ }^{\mathrm{h}, \mathrm{i}}$} & \multirow{2}{*}{$\begin{array}{c}\text { Day } 4 \\
( \pm 1 \text { day })\end{array}$} & \multirow{2}{*}{$\begin{array}{c}\text { Day } 8 \\
( \pm 2 \text { days })\end{array}$} & \multirow{2}{*}{$\begin{array}{c}\text { Day } 14 \\
(-4 \text { days })^{g}\end{array}$} & \multirow{2}{*}{$\begin{array}{c}\text { Day } 28 \\
( \pm 2 \text { days })^{\mathrm{e}}\end{array}$} & \multirow{2}{*}{$\begin{array}{c}\text { Day } 42 \\
\text { ( } \pm 3 \text { days })\end{array}$} \\
\hline & & & Pre & Post $^{d}$ & & & & & \\
\hline \multicolumn{2}{|c|}{ Informed Consent Form } & $\mathrm{X}$ & & & & & & & \\
\hline \multicolumn{10}{|c|}{ Eligibility } \\
\hline \multicolumn{2}{|c|}{ Inclusion and Exclusion Criteria ${ }^{\mathrm{a}}$} & $\mathrm{X}$ & $\mathrm{X}$ & & & & & & \\
\hline \multicolumn{10}{|c|}{ Baseline Characteristics } \\
\hline \multicolumn{2}{|c|}{ Medical History } & $\mathrm{X}$ & $\mathrm{X}$ & & & & & & \\
\hline \multicolumn{2}{|c|}{ Concomitant Medications } & $\mathrm{X}$ & $\mathrm{X}$ & & $\mathrm{X}$ & $\mathrm{X}$ & $\mathrm{X}$ & $\mathrm{X}$ & \\
\hline \multicolumn{2}{|c|}{ Demographics } & $\mathrm{X}$ & & & & & & & \\
\hline \multicolumn{10}{|c|}{ Safety Assessments } \\
\hline \multicolumn{2}{|c|}{ Physical Examination } & $\mathrm{X}$ & $\mathrm{X}$ & & & & $\mathrm{X}$ & $\mathrm{X}$ & \\
\hline \multicolumn{2}{|c|}{ Weight $(\mathrm{kg})$} & $\mathrm{X}$ & $\mathrm{X}$ & & $\mathrm{X}$ & $\mathrm{X}$ & $X$ & $\mathrm{X}$ & \\
\hline \multicolumn{2}{|l|}{ Vital Signs $^{\mathrm{b}}$} & $\mathrm{X}$ & $\mathrm{X}$ & $X^{d}$ & $X^{d}$ & $X^{d}$ & $X^{d}$ & $\mathrm{X}$ & \\
\hline \multicolumn{2}{|c|}{ Safety Laboratory Measures ${ }^{\mathrm{c}}$} & $\mathrm{X}$ & $\mathrm{X}$ & $X^{d}$ & $X^{d}$ & $X^{d}$ & $X^{d}$ & $\mathrm{X}$ & \\
\hline \multicolumn{2}{|c|}{ Urine Pregnancy Test ${ }^{\mathrm{f}}$} & $\mathrm{X}$ & $\mathrm{X}$ & & & & & $\mathrm{X}$ & \\
\hline \multicolumn{2}{|c|}{ Bio-impedance Analysis } & $X^{j}$ & $X^{j}$ & & & & $\mathrm{X}$ & $\mathrm{X}$ & \\
\hline \multicolumn{2}{|c|}{ Transthoracic Doppler Echocardiogram } & $\mathrm{X}^{\mathrm{k}}$ & $\mathrm{X}^{\mathrm{k}}$ & & & & $\mathrm{X}$ & $\mathrm{X}$ & \\
\hline \multicolumn{2}{|l|}{$\mathrm{CPX}$} & $\mathrm{X}^{\mathrm{j}}$ & $\mathrm{X}^{\mathrm{j}}$ & & & & $\mathrm{X}$ & $\mathrm{X}$ & \\
\hline 12-lead ECG & & & $\mathrm{X}$ & $X^{d}$ & $X^{d}$ & $X^{d}$ & & & \\
\hline Adverse Eve & & & $A d v$ & nts will & prded via st & diary and re & ved at each $s$ & visit & \\
\hline Follow-up Te & & & & & & & & & $\mathrm{X}$ \\
\hline Quality of L & & & & & & & & & \\
\hline Kansas City & naire & $\mathrm{X}^{\mathrm{j}}$ & $\mathrm{X}^{\mathrm{j}}$ & & & & $\mathrm{X}$ & $\mathrm{X}$ & \\
\hline Duke Activit & & $\mathrm{X}^{\mathrm{j}}$ & $\mathrm{X}^{\mathrm{j}}$ & & & & $\mathrm{X}$ & $\mathrm{X}$ & \\
\hline Brief Pain In & & & $\mathrm{X}$ & & $\mathrm{X}$ & $\mathrm{X}$ & $\mathrm{X}$ & $\mathrm{X}$ & \\
\hline Pharmacoki & nics Ass & sments & & & & & & & \\
\hline Blood draw $\mathrm{f}$ & & & $\mathrm{X}$ & $X^{d}$ & $X^{d}$ & $X^{d}$ & $X^{d}$ & $X$ & \\
\hline Dispensatior & terials & & & & & & & & \\
\hline $\begin{array}{l}\text { Investigation } \\
\text { Returned (R) }\end{array}$ & & & $\mathrm{D}$ & & $\mathrm{R} / \mathrm{D}$ & $\mathrm{R} / \mathrm{D}$ & $\mathrm{R}^{\mathrm{e}}$ & & \\
\hline
\end{tabular}




\begin{tabular}{|c|c|c|c|c|c|c|c|c|}
\hline Trial Period: & \multicolumn{2}{|c|}{ Eligibility } & \multicolumn{4}{|c|}{ Treatment } & \multicolumn{2}{|c|}{ Follow-up } \\
\hline \multirow{2}{*}{ Assessment } & \multirow{2}{*}{$\begin{array}{c}\text { Screening } \\
(\text { Day -28 to } 1)^{\mathrm{h}}\end{array}$} & \multicolumn{2}{|c|}{ Baseline (Day 1) ${ }^{\mathrm{h}, \mathrm{i}}$} & \multirow{2}{*}{$\begin{array}{c}\text { Day } 4 \\
( \pm 1 \text { day })\end{array}$} & \multirow{2}{*}{$\begin{array}{c}\text { Day } 8 \\
( \pm 2 \text { days })\end{array}$} & \multirow{2}{*}{$\begin{array}{c}\text { Day } 14 \\
(-4 \text { days })^{g}\end{array}$} & \multirow{2}{*}{$\begin{array}{c}\text { Day } 28 \\
( \pm 2 \text { days })^{\mathrm{e}}\end{array}$} & \multirow{2}{*}{$\begin{array}{c}\text { Day } 42 \\
( \pm 3 \text { days })\end{array}$} \\
\hline & & Pre & Post $^{d}$ & & & & & \\
\hline Study Diary I & $\mathrm{D}$ & $\mathrm{R} / \mathrm{D}$ & & $\mathrm{R} / \mathrm{D}$ & $\mathrm{R} / \mathrm{D}$ & $\mathrm{R} / \mathrm{D}$ & $\mathrm{R}$ & \\
\hline \multicolumn{9}{|l|}{ Dosing } \\
\hline IP Administe & & & \multicolumn{4}{|c|}{ 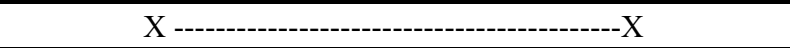 } & & \\
\hline Suggested Co & & & \multicolumn{4}{|c|}{$\begin{array}{l}\text { It is suggested (not required) that site staff contact subjects by } \\
\text { phone approx. every } 1-2 \text { days to confirm dosing compliance }\end{array}$} & & \\
\hline
\end{tabular}

a Inclusion and Exclusion Criteria: all criteria should be met at Baseline visit

b Vital Signs: pulse, 10-minute resting blood pressure, temperature, respiration rate

c Safety Laboratory Measures: chemistry, hematology and cardiac biomarkers will be performed each time (at the same time as PK/PD blood draws). Urine Pregnancy Test will be completed at Screening, Baseline (pre-dose) and Day 28 visits, if applicable

d Post-dose Assessments: Safety ECG, vital signs, and safety laboratory measures and PK/PD blood draws to be completed at 1 hour ( \pm 15 min) after investigational product administration. At the Baseline (Day 1; post-dose) visit, for safety laboratory measures, only hsCRP (a PD biomarker analyzed as part of safety labs: chemistry) is measured.

e Early Termination: if a subject is an Early Termination (i.e., discontinues before Day 28 visit is completed), the Day 28 visit assessments (plus drug reconciliation) should be completed at the Early Termination visit.

f Urine Pregnancy Test: women of child-bearing potential only.

g Investigational Product Administered: will occur under supervision of site personnel on all study treatment days involving a site visit beginning at Baseline (Day 1). Subjects will self-administer all other doses of investigational product. Subjects in cohort 1 should administer investigational product at approximately the same time each day (daily dose); subjects in cohort 2 should administer investigational product at approximately the same time in the morning (first dose) and in the afternoon (second dose) each day; subjects in cohort 3 should administer investigational product a approximately the same time in the morning (first dose), mid-day (second dose), afternoon (third dose) and in the evening (fourth dose). The dose administered at the Day 14 visit will be the last administered dose of investigational product. The Day 14 visit will be scheduled to achieve as many treatment days as possible (up to the maximum of 14 days).

h Screening and Baseline visits: the Screening and Baseline visits may be combined into a single visit. In this case, all Screening and Baseline procedures should be performed and recorded as the Baseline visit. Available medical data collected/performed within 72 hours prior to randomization may be used to satisfy study procedures.

i Baseline visit: procedures performed at the Screening visit that are completed within 72 hours prior to randomization may be used to satisfy Baseline study procedures.

j Bio-impedance Analysis, CPX and Quality of Life Assessments: considered optional during the Screening visit. If completed during the Screening visit, these assessments do not need to be repeated if the Baseline (Day 1; pre-dose) visit is $<72$ hours from Screening visit.

k Transthoracic Doppler Echocardiogram: this assessment is considered optional during the Screening visit if a previous qualifying TTE (LVEF $<40 \%$ ) has been completed during the past 6 months. If TTE is completed during the Screening visit and the Screening visit and Baseline (Day 1; pre-dose) visit occur within 72 hours, then TTE does not need to be repeated at the Baseline (Day 1; predose) visit. 


\section{ADVERSE EVENTS}

An adverse event (AE) can be any unfavorable, unintended sign (including an abnormal laboratory finding, for example), symptom or disease temporally associated with the use of the investigational product, whether or not considered related to the medicinal product.

It is the responsibility of the Investigator to document all AEs that occur during the course of the trial. Adverse events should be documented as a single medical diagnosis. When this is not possible, the AE should be documented in terms of signs and/or symptoms observed by the Investigator or reported by the subject at each trial visit.

Adverse events occurring after the signing of the ICF will be recorded. The Investigator will follow subjects with any adverse events until one of the following conditions occurs:

- $\mathrm{AE}$ is stabilized and/or resolved

- $\quad$ Subject is lost to follow-up

All AEs will be classified by intensity according to Table 8.1, and guidelines for determining the relationship between any $\mathrm{AE}$ and the investigational product will be according to Table 8.2. If the relationship between an adverse event and the investigational product is classified as "related", "probable" or "possible, the event will be regarded as a reaction to the investigational product.

\section{Table 8.1 Classification of Adverse Events by Intensity}

\section{Grade 1 (MILD):}

The symptom is barely noticeable to the subject and does not influence performance or functioning. Allowed concomitant medication is not ordinarily indicated for relief of mild AEs.

Examples specific to the trial would include: Transient, barely noticeable headache

\section{Grade 2 (MODERATE):}

The symptom is of sufficient severity to make the subject uncomfortable and to influence performance of daily activities. Allowed concomitant medication may be indicated for relief of moderate AEs.

Examples specific to the trial would include: Dull persisting headache.

\section{Grade 3 (SEVERE):}

The symptom causes severe discomfort, sometimes of such severity that the subject cannot continue in the trial. Daily activities are significantly impaired or prevented by the symptom. Allowed concomitant medication may be indicated for relief of severe AEs.

Examples specific to the trial would include: Excruciating migraine. 
Table 8.2 Guidelines for Determining the Relationship Between Any Adverse Event and the Investigational Product

\begin{tabular}{|l|l|}
\hline Related: & $\begin{array}{l}\text { There is a reasonable causal relationship between the investigational product } \\
\text { administration and the adverse event. The event responds to withdrawal of } \\
\text { investigational product (dechallenge), and recurs with rechallenge when clinically } \\
\text { feasible. }\end{array}$ \\
\hline Probable: & $\begin{array}{l}\text { There is a reasonable temporal sequence from investigational product administration. } \\
\text { The event abates upon discontinuation of the investigational product, and cannot be } \\
\text { reasonably explained by the known characteristics of the subject's clinical state. }\end{array}$ \\
\hline Possible: & $\begin{array}{l}\text { The event may or may not follow a reasonable temporal sequence from investigational } \\
\text { product administration. The event could have been produced or mimicked by the } \\
\text { subject's clinical state or by other modes of therapy concomitantly administered to the } \\
\text { subject. }\end{array}$ \\
\hline Unlikely: & $\begin{array}{l}\text { There is no reasonable temporal association between the investigational product } \\
\text { administration and the event. The event could have been produced by the subject's } \\
\text { clinical state or by other modes of therapy administered to the subject. }\end{array}$ \\
\hline Unrelated: & $\begin{array}{l}\text { There is not a temporal relationship to investigational product administration or there } \\
\text { is a reasonable causal relationship between another therapy, concurrent disease, or } \\
\text { circumstance and the event. }\end{array}$ \\
\hline
\end{tabular}

\subsection{SERIOUS ADVERSE EVENTS}

An SAE or serious adverse event is defined as any untoward medical occurrence that:

- Results in death;

- Is immediately life-threatening, (the term "life-threatening" in the definition of "serious" refers to an event in which the subject was at risk of death at the time of the event; it does not refer to an event which hypothetically might have caused death if it were more severe);

- Requires in-patient hospitalization or prolongation of existing hospitalization (hospitalization for elective surgery for a baseline condition is not considered an AE);

- Results in persistent or significant disability/incapacity (permanent or substantial disruption of a person's ability to conduct normal life functions);

- Is a congenital anomaly/birth defect; or

- Is a medically important event that may not be immediately life-threatening or result in death or hospitalization, but may jeopardize the subject and may require medical or surgical intervention to prevent one of the above listed outcomes. 


\subsection{UNEXPECTED ADVERSE EVENT}

An unexpected adverse event is defined as any adverse drug experience (i.e., untoward or unintended response) that has not been previously observed (e.g., included in the Investigator's Brochure).

\subsection{SUSPECTED UNEXPECTED SERIOUS ADVERSE REACTIONS (SUSARS)}

Unexpected adverse reactions are suspected unexpected serious adverse reactions if the following three conditions are met:

1. The event must be serious (see Section 8.1);

2. There must be a certain degree of probability that the event is a harmful and an undesirable reaction to the medicinal product under investigation, regardless of the administered dose; and

3. The adverse reaction must be unexpected (see Section 8.2).

\subsection{ADVERSE EVENTS PROCEDURES}

The Investigator should obtain any information regarding the occurrence of adverse events through open-ended questioning of the subject, physical examination and review of laboratory results.

All adverse events, whether serious or not, will be recorded in the Adverse Event page of the subject's CRF and any other related documents.

Information to be described for any adverse event includes:

- Date of onset of the AE

- Medical diagnosis of the AE (if a medical diagnosis cannot be determined, a description of each sign or symptom characterizing the event should be recorded). Action taken for the AE, such as: none; change in the investigational product administration (e.g., temporary interruption in dosing); drug treatment required; non-drug treatment required; hospitalization or prolongation of hospitalization required; diagnostic procedure performed, subject discontinued from the trial

- Outcome of the AE, such as: subject recovered without sequelae; subject recovered with sequelae; event ongoing; subject died (notify the Sponsor immediately, and complete the Serious Adverse Event page and the Final Visit section of the CRF)

- Date of resolution of the AE and whether or not it was an SAE 


\subsubsection{Reporting Serious Adverse Events (SAEs)}

Full details regarding the collection and reporting procedures for this study are contained in the Safety Management Plan for Study OLT1177-06.

\subsubsection{Reporting SAEs to the Sponsor}

Any fatal or life-threatening serious adverse events must be reported to the Medical Monitor immediately when known by phone, e-mail or the study safety web portal. Any other serious adverse events must be reported to the Medical Monitor within 24 hours of knowledge by phone, e-mail or the study safety web portal. A full description of procedures for the reporting of SAEs is contained in the Safety Management Plan.

Within 24 hours of knowledge, the Serious Adverse Event Form must be sent to the Medical Monitor whether full information regarding the event is known or not. If full information is not known, additional follow-up by the Investigator will be required.

All SAEs occurring by Day 42 ( \pm 3 day) of the study (28 days after the last dose of investigational product) must be reported to the Sponsor.

\subsubsection{Contact Information for Reporting Serious Adverse Event}

Investigator will report any serious adverse events to Medical Monitor, as follows:

Curtis Scribner, MD

4287 Howe Street

Oakland, CA 94611

Main Tel: +1 833-652-8321 x108

Mobile Tel: +1 510-914-8368

Fax: +1 510-985-1665

E-mail: curt@clscribs.com and operations@olatec.com

\subsubsection{Reporting Pregnancies}

Any pregnancies occurring during the study must be reported to the Sponsor within one business day of the clinical site becoming aware of it. Initial and follow-up pregnancy reporting will be performed in accordance with the Safety Management Plan for Study OLT1177-06. All pregnancies will be followed through birth. 


\section{STATISTICAL METHODS}

\subsection{GENERAL CONSIDERATIONS}

\subsubsection{Determination of Sample Size}

An assessment of sample size or power analyses is not applicable for this Phase $1 \mathrm{~b}$ study, as the goal of the study is to characterize any side effects following reasonable exposure to the drug.

Cohort 1 will be comprised of 8 subjects who will receive $500 \mathrm{mg}$ daily of investigational product; Cohort 2 will include 8 additional subjects who will receive $500 \mathrm{mg}$ twice daily; and Cohort 3 will include 8 additional subjects who will receive $500 \mathrm{mg}$ four times daily. Each cohort will also enroll 2 subjects who will receive Placebo Capsules for a total of 10 subjects enrolled into each cohort. A sample size of 24 subjects receiving dapansutrile capsules (combining Cohorts 1,2 and 3) would provide a power of $90 \%$ to exclude a difference in $>25 \%$ of any safety parameter (see Reasons for Interruption of the Investigational Product in the Individual Subject), with an estimated standard deviation of $30 \%$.

For the pharmacodynamics analysis, the sample size of 24 subjects on investigational product would also provide a $>80 \%$ power to detect a 35\% reduction in hsCRP (e.g., from 6 to $3.9 \mathrm{mg} / \mathrm{L}$; estimated standard deviation $2 \mathrm{mg} / \mathrm{L}$ ) and a $>99 \%$ for a reduction that exceeds $50 \%$.

\subsubsection{Statistical and Analytical Plans}

\subsubsection{Study Populations}

Two populations will be defined for this study: 1) the Intent-to-Treat Population will consist of all subjects who have taken at least one dose of investigational product; 2) the Per-Protocol Population will consist of all subjects who take $80 \%$ or more of the total expected doses of investigational product and have no major protocol violations as determined by the Medical Monitor in consultation with the Principal Investigator.

\subsubsection{Planned Statistical Analyses}

Baseline measurements and demographic characteristics will be summarized using descriptive statistics with median and interquartile range for each Cohort and with data from all cohorts combined. Descriptive summaries of categorical measurements will consist of frequencies and proportions.

The summaries for each measurement will be provided at each visit and analyzed with the nonparametric Wilcoxon or Friedman test for paired data pooled for the three cohorts for 2 or more time points, respectively. The study will be underpowered to detect differences between 
Cohorts 1, 2 and 3, any comparisons between the three groups will be viewed as hypothesisgenerating only.

The Statistical Analysis Plan (SAP) for Study OLT1177-06 contains a complete description of all populations and analyses to be performed, including a description of the procedures for accounting for missing, unused and spurious data and for reporting any deviation from the SAP.

\section{REGULATORY AND LEGAL OBLIGATIONS}

\subsection{PROTOCOL AMENDMENTS}

The Sponsor will immediately inform the IRB and Principal Investigators of any required protocol changes, such as to eliminate any hazard to a subject. Changes to the protocol must be in the form of a written amendment prepared and approved by Sponsor. Such changes will require the written approval of the IRB and will only be implemented following receipt of such approval. The Sponsor, as required by local regulation, will also submit all amendments to local regulatory authorities. Amendment to the protocol may also require Sponsor to revise the ICF. Such a revised ICF must then be submitted to the IRB for review and approval; the revised ICF must be used to obtain consent from subjects currently randomized/enrolled in the trial if they are affected by the amendment and to obtain consent from new subjects prior to enrollment.

\subsection{MONITORING}

Qualified representative(s) of the Sponsor or Sponsor designees, "study monitor(s)," will monitor the trial according to a predetermined monitoring plan.

The Investigator must allow the study monitor(s) to periodically review, at mutually convenient times, during the trial and after the trial has been completed, all CRFs and office, hospital, and laboratory records supporting the participation of each subject in the trial. The CRFs and other documentation supporting the trial must be kept up-to-date by the Investigator and the site personnel at the investigative site. The Investigator will ensure that the study monitor or other qualified representatives of the Sponsor are given access to all study-related documents and has adequate time and space to conduct the monitoring visit including availability of the Investigator and site personnel to discuss findings.

The study monitor will review the various records of the trial (CRFs, subject medical and laboratory records, and other source documents). The study monitor will verify the CRF data against original source documentation for accuracy and completeness. The study monitor will identify data discrepancies and collaborate with the Investigator and site personnel to resolve the discrepancies in a timely manner. Protocol deviations will also be identified and recorded 
on a "Protocol Deviation Log." The study monitor will ensure that each issue identified during a monitoring visit is appropriately documented, reported, and resolved in a timely manner.

In addition to the above, Representatives of the Sponsor's auditing staff or government inspectors may review the conduct/results of the trial at the site. The Investigator must promptly notify the Sponsor of any audit requests by regulatory authorities.

\subsection{PRE-TRIAL DOCUMENTATION}

Prior to initiating the trial, the Sponsor will obtain the following documentation:

- A signed FDA Form 1572

- A current (within 2 years), dated and signed curriculum vitae for the Principal Investigator and each Sub-Investigator listed on the FDA Form 1572

- A copy of the medical license from the state in which the trial is being conducted for the Principal Investigator

- A letter from the IRB stipulating approval of the protocol, the ICF and any other material provided to potential trial subjects with information about the trial (e.g., advertisements)

- A copy of the IRB-approved ICF

- A Protocol Agreement signed by the Principal Investigator

- A completed Financial Disclosure Form for the Principal Investigator and any other persons listed on the 1572

- An Investigators Brochure Acknowledgement signed by the Principal Investigator

Additionally, prior to initiating the trial, the Sponsor will obtain copies of the following documentation from the central laboratory:

- Current IRB membership list for IRB's without a multiple project assurance number or an IRB organization number under the Federal Wide Assurance program (www.ohrp.osophs.dhhs.gov)

- Current laboratory certification for the reference laboratory

- A list of current laboratory normal values for the reference laboratory

\subsection{SUBJECT CONFIDENTIALITY AND DISCLOSURE}

It is the responsibility of the Principal Investigator to ensure that the confidentiality of all subjects participating in the trial and all of their medical information is maintained at all times prior to, during and following completion of the trial. CRFs and all other documents will 
identify each subject by SID. All CRFs and any identifying information must be kept in a secure location with access limited to designated site personnel.

Personal medical information may be reviewed by the study monitor or other qualified representatives of the Sponsor, the IRB or regulatory authorities in the course of monitoring the progress of the trial. Every reasonable effort will be made to maintain such information as confidential.

The results of the trial may be presented in reports, published in scientific journals or presented at medical meetings in consultation with the Sponsor and Principal Investigator in accordance with applicable law (see Section 10.7); however, in all cases no reference will be made to any subject by name.

\subsection{SOURCE DOCUMENTS}

The Principal Investigator will maintain trial-related source documents, separate from the CRFs, including: clinical charts, medical records, original laboratory, radiology and pathology reports, pharmacy records, etc. The Principal Investigator will document the name and number of the trial and the date on which the subject signed the ICF in the clinic chart or medical record. Source documents will contain a complete description of each subject's medical care, and must be available for source document verification against entries in the CRFs when the Project Manager or study monitor visits the site. All information obtained from source documents will be kept in strict confidentiality.

\subsection{RECORD RETENTION}

Site shall maintain all such information until the earlier of (i) two (2) years after the first regulatory approval of the investigational product by the FDA, EMA or another regulatory authority or (ii) two (2) years after Sponsor has notified Site and the Principal Investigator that clinical development of the investigational product has been discontinued, or (iii) 15 years, but in any event for the full duration required by Applicable Laws. Site shall not destroy any such information without the prior written permission of Sponsor. Trial records that must be retained include copies of: CRFs, signed ICFs, correspondence with the IRB, investigational product dispensing and inventory records, source documents, clinic charts, medical records, laboratory results, radiographic reports and screening/enrollment logs.

Should the Principal Investigator relocate or retire, or should there be any changes in the archival arrangements for the trial records, the Sponsor must be notified. The responsibility for maintaining the trial records may be transferred to another suitable individual, but the Sponsor must be notified of the identity of the individual assuming responsibility for maintaining the trial records and the location of their storage. If no other individual at the site 
is willing to assume this responsibility, the Sponsor will assume responsibility for maintaining the trial records.

\subsection{PUBLICATION POLICY}

All information and data obtained in the course of the trial are the property of the Sponsor and are considered confidential. Publication of information and data will be determined in consultation with the Sponsor and Principal Investigator, and in accordance with applicable law and the study Clinical Trial Agreement.

\section{REFERENCES}

1. Abbate, A, Salloum FN, Vecile E, et al. Anakinra, a recombinant human interleukin-1 receptor antagonist, inhibits apoptosis in experimental acute myocardial infarction. Circulation 2008;117:2670-2683.

2. Abbate A, Kontos MC, Grizzard JD, et al. Interleukin-1 blockade with anakinra to prevent adverse cardiac remodeling after acute myocardial infarction (Virginia Commonwealth University Anakinra Remodeling Trial [VCU-ART] Pilot Study). Am J Cardiol 2010;105:1371-1377.

3. Abbate A, Salloum FN, Van Tassell BW, et al. Alterations in the interleukin-1/interleukin1 receptor antagonist balance modulate cardiac remodeling following myocardial infarction in the mouse. PLOS ONE 2011;6(11):e27293.

4. Abbate A, Van Tassell BW, Biondi-Zoccai GG. Blocking interleukin-1 as a novel therapeutic strategy for secondary prevention of cardiovascular events. BioDrugs 2012;26(4):217-233.

5. Abbate A, Van Tassell BW, Biondi-Zoccai G, et al. Effects of interleukin-1 blockade with anakinra on adverse cardiac remodeling and heart failure after acute myocardial infarction [from the Virginia Commonwealth University-Anakinra Remodeling Trial (2) (VCUART2) Pilot Study]. Am J Cardiol 2013;111:1394-1400.

6. Abbate A, Kontos MC, Abouzaki NA, et al. Comparative safety of interleukin-1 blockade with anakinra in patients with ST-segment elevation acute myocardial infarction (from the VCU-ART and VCU-ART2 Pilot Studies). Am J Cardiol 2015;115:288-292.

7. Bujak M, Dobaczewski M, Chatila K, et al. Interleukin-1 receptor type 1 signaling critically regulates infarct healing and cardiac remodeling. Am J Pathol 2008;173(1):57-67.

8. Dinarello CA. Interleukin-1 in the pathogenesis and treatment of inflammatory diseases. Blood 2011;117(14):3720-3732. 
9. Duncan JA, Bergstralh DT, Wang Y, Willingham SB, Ye Z, Zimmerman AG, Ting JPY. Cryopyrin/NALP3 binds ATP/dATP, is an ATPase, and requires ATP binding to mediate inflammatory signaling. PNAS 2007;104(19):8041-8046.

10. Forman DE, Arena R, Boxer R, et al. Prioritizing functional capacity as a principal end point for therapies oriented to older adults with cardiovascular disease. Circulation 2017;135(16):e894-e918.

11. Fletcher GF, Ades PA, Kligfield P, Arena R, Balady GJ, Bittner VA, Coke LA, Fleg JL, Forman DE, Gerber TC, Gulati M. Exercise standards for testing and training. Circulation 2013 Aug 20;128(8):873-934.

12. Harouki N, Nicol L, Remy-Jouet I, et al. The IL-1 $\beta$ antibody gevokizumab limits cardiac remodeling and coronary dysfunction in rats with heart failure. J Am Coll Cardiol Basic Trans Science 2017;2(4):418-430.

13. He Y, Varadarajan S, Muñoz-Planillo R, Burberry A, Nakamura Y, Núñez G. 3,4-Methylene- $\beta$-nitrostyrene inhibits NLRP3 inflammasome activation by blocking assembly of the inflammasome $J$ Biol Chem 2014;289(2):1142-1150.

14. Juliana C, Fernandes-Alnemri T, Wu J, Datta P, Solorzano L, Yu J-W, Meng R, Quong AA, Latz E, Schott CP, Alnemri ES. Anti-inflammatory compound parthenolide and Bay 11-7082 are direct inhibitors of the inflammasome. J Biol Chem 2010;285(13):9792-9802.

15. Kawaguchi M, Takahashi M, Hata $\mathrm{T}$, et al. Inflammasome activation of cardiac fibrosis is essential for myocardial ischemia/reperfusion injury. Circulation 2011;123:594-604.

16. Kumar A, Thota V, Dee L, Olson J, Uretz E, Parrillo JE. Tumor necrosis factor alpha and interleukin $1 \beta$ are responsible for in vitro myocardial cell depression induced by human septic shock serum. $J$ Exp Med 1996;183:949-958.

17. Latz E, Xiao TS, Stutz A. Activation and regulation of the inflammasome. Nat Rev Immunol 2013;13(6):397-411.

18. Libby P, Theroux P. Pathophysiology of coronary artery disease. Circulation 2005;111:3481-3488.

19. Marchetti C, Chonjnacki J, Toldo S, et al. A novel pharmacological inhibitor of the NLRP3 inflammasome limits myocardial injury following ischemia-reperfusion in the mouse. $J$ Cardiovasc Pharmacol 2014;63(4):316-322.

20. Marchetti C, Toldo S, Chojnacki J, et al. Pharmacologic inhibition of the NLRP3 inflammasome preserves cardiac function after ischemic and non-ischemic injury in the mouse. J Cardiovasc Pharmacol 2015;66(1):1-8.

21. Marchetti C, Swartzwelter B, Gamboni F, et al. OLT1177, a $\beta$-sulfonyl nitrile compound safe in humans, inhibits the NLRP3 inflammasome and reverses the metabolic cost of inflammation. Proc Nat Acad Sci. USA 2018; accepted for publication. 
22. Mason JC, Libby P. Cardiovascular disease in patients with chronic inflammation: mechanisms underlying premature cardiovascular events in rheumatologic conditions. Eur Heart J 2015;36:482-489.

23. Mezzaroma E, Toldo S, Farkas D, et al. The inflammasome promotes adverse cardiac remodeling following acute myocardial infarction in the mouse. Proc Natl Acad Sci 2011;108(49):19725-19730.

24. Pickering TG, Hall JE, Appel LJ, Falkner BE, Graves J, Hill MN, et al.; Subcommittee of Professional and Public Education of the American Heart Association Council on High Blood Pressure Research. Recommendations for blood pressure measurement in humans and experimental animals. Part 1: blood pressure measurement in humans. Hypertension $2005 ; 45: 142-61$.

25. Sandanger O, Ranheim T, Vinge LE, et al. The NLRP3 inflammasome is up-regulated in cardiac fibroblasts and mediates myocardial ischaemia-reperfusion injury. Cardiovasc Res 2013;99:164-174.

26. Seropian IM, Toldo S, Van Tassell BW, Abbate A. Anti-inflammatory strategies for ventricular remodeling following ST-segment elevation acute myocardial infarction. $J \mathrm{Am}$ Coll Cardiol 2014;63(16):1593-1603.

27. Toldo S, Seropian IM, Mezzaroma E, et al. Alpha-1 antitrypsin inhibits caspase-1 and protects from acute myocardial ischemia-reperfusion injury. J Molec Cell Cardiol 2011;51:244-251.

28. Toldo S, Mezzaroma E, Van Tassell BW, et al. Interleukin-1 $\beta$ blockade improves cardiac remodeling after myocardial infarction without interrupting the inflammasome in the mouse. Exp Physiol 2013;98.3:734-745.

29. Toldo S, Mezzaroma E, O'Brien L, et al. Interleukin-18 mediates interleukin-1-induced cardiac dysfunction. Am J Physiol Heart Circ Physiol 2014;306:H1025-H1031.

30. Toldo S, Mezzaroma E, Mauro AG, et al. The inflammasome in myocardial injury and cardiac remodeling. Antioxidant Redox Sig 2015;22(13):1146-1161.

31. Toldo S, Mauro AG, Marchetti C, et al. Novel NLRP3 inflammasome inhibitor OLT1177 reduces infarct size in a mouse model of myocardial ischemia reperfusion injury. Circulation 2017; 136 Suppl 1: A18066.

32. Toldo S, Mauro AG, Cutter Z, et al. The NLRP3 Inflammasome inhibitor, OLT1177 (Dapansutrile), reduces infarct size and preserves contractile function after ischemia reperfusion injury in the mouse. J Cardiovasc Pharmacol 2019 Feb 8; Volume Publish Ahead of Print; doi: 10.1097/FJC.0000000000000658.

33. Van Tassell BW, Arena RA, Toldo S et al. Enhanced interleukin-1 activity contributes to exercise intolerance in patients with systolic heart failure. PLoS ONE 2012;7(3):e33438. 
34. Van Tassell BW, Toldo S, Mezzaroma E, Abbate A. Targeting interleukin-1 in heart disease. Circulation 2013a;128:1910-1923.

35. Van Tassell BW, Seropian IM. Interleukin-1 $\beta$ induces a reversible cardiomyopathy in the mouse. Inflamm Res 2013b;62:637-640.

36. Van Tassell BW, Arena R, Biondi-Zoccai G, et al. Effects of inteleukin-1 blockade with anakinra on aerobic exercise capacity in patients with heart failure and preserved ejection fraction (from the D-HART Pilot Study). Am J Cardiol 2014;113(2):321-327.

37. Van Tassell BW, Abouzaki NA, Erdle CO, et al. Interleukin-1 blockade in acute decompensated heart failure: A randomized, double-blinded, placebo-controlled pilot study. J Cardiovasc Pharmacol 2016;67:544-551.

38. Van Tassell BW, Canada J, Carbone S, et al. Interleukin-1 blockade in recently decompensated systolic heart failure: the recently decompensated heart failure anakinra response trial (REDHART). Eur J Heart Fail 2017;19 Suppl S1:368.

39. Wasserman K, Hansen JE, Sue DY, Casaburi R, Whipp BJ. Principles of Exercise Testing and Interpretation: Including Pathophysiology and Clinical Applications. 3rd edition. Philadelphia, Pa, USA: Lippincott, Williams \& Wilkins; 1999. 


\section{APPENDIX 1 DETAILED DESCRIPTION OF STUDY DAYS}

\section{CLINICAL VISITS}

\subsection{Screening Visit (Day -28 to 1)}

The Screening visit will take place within the 28 days prior to randomization (Day -28 to 1 ) unless otherwise approved by the Medical Monitor or designee. During the Screening visit, trial procedures included in the ICF will be explained in detail by designated site personnel. Informed consent will be obtained from all screened subjects. All subjects will be given ample time to review the informed consent form and ask questions. A signed ICF must then be obtained prior to any trial procedures being performed.

The Screening and Baseline visits may be combined into a single visit, in which case all Screening and Baseline procedures should be performed and recorded as the Baseline visit.

The following evaluations and procedures will be performed at this visit:

- Informed Consent: review, explanation and signature on ICF

- Demographics: race, gender, age (years)

- Review of inclusion/exclusion criteria

- Medical history: A full medical history, including medication history, will be taken and all concomitant medications/therapies taken prior to Screening should be recorded on the concomitant medications/therapies source document and CRF page

- Physical examination (including height $(\mathrm{cm})$ and weight $(\mathrm{kg})$ measurements)

- Vital signs (pulse, resting blood pressure [must be resting for 10 minutes], respiration rate, and temperature)

- Blood samples for screening safety laboratory measures (hematology, chemistry and cardiac biomarkers)

- Urine pregnancy test for all females of childbearing potential

- Bio-impedance Analysis (optional)

- Transthoracic Doppler Echocardiography (before CPX) (optional if a previous qualifying TTE $[\mathrm{LVEF}<40 \%]$ has been completed during the past 6 months)

- $\mathrm{CPX}$ (optional)

- Quality of Life Assessments (optional)

- Kansas City Cardiomyopathy Questionnaire

- Duke Activity Status Index 


\subsection{Baseline Visit (Day 1)}

\subsubsection{Prior to Drug Administration}

The following evaluations and procedures will be performed at this visit:

- Review of inclusion/exclusion criteria

- Review of any changes to medical history

- Review of any changes to concomitant medications/therapies

- Urine pregnancy test for all females of childbearing potential

- Physical examination (including weight ( $\mathrm{kg}$ ) measurement)

- Vital signs (pulse, resting blood pressure [must be resting for 10 minutes], respiration rate, and temperature)

- Bio-impedance Analysis (if not performed within 72 hours prior at Screening visit)

- Transthoracic Doppler Echocardiography (before CPX) (if not performed within 72 hours prior at Screening visit)

- $\mathrm{CPX}$ (if not performed within 72 hours prior at Screening visit)

- Quality of Life Assessments (if not performed within 72 hours prior at Screening visit) and Pain Assessment

- Kansas City Cardiomyopathy Questionnaire

- Duke Activity Status Index

- Brief Pain Index

- Blood samples for safety laboratory measures (hematology, chemistry and cardiac biomarkers) and PK/PD analysis

- 12-lead ECG

The Screening and Baseline visits may be combined into a single visit, in which case all Screening and Baseline procedures should be performed and recorded as the Baseline visit. In addition to the procedures outlined above, the following screening procedures should be performed if the Screening and Baseline visits are combined:

- Informed Consent: review, explanation and signature on ICF (prior to performance of any study procedures)

- Demographics: race, gender, age (years) 
- Medical history: A full medical history, including medication history and all concomitant medications/therapies taken prior to Baseline should be recorded on the concomitant medications/therapies source document and CRF page

- Height (cm) (as part of physical examination)

The Principal Investigator or designated Sub-Investigator will only enroll subjects who meet all protocol eligibility criteria.

\subsubsection{Drug Administration}

Subjects meeting all entry criteria will have the following procedures performed:

- Dispense investigational product

- Dispense Study Diary including Dosing Diary and subject instructions

- Administration of investigational product under supervision of site personnel

\subsubsection{Post Drug Administration}

Following first administration of investigational product, subjects will remain at the study clinic for approximately 1 to 2 hours at which time the following assessments will be completed:

- Assessment of adverse events

- At 1 hour ( $\pm 15 \mathrm{~min})$ after investigational product administration:

- Vital signs (pulse, resting blood pressure [must be resting for 10 minutes], respiration rate, and temperature)

- Blood sample for PK/PD analysis including hsCRP (a PD biomarker analyzed as part of safety labs: chemistry)

- 12-lead ECG

\subsection{Day 4 Visit ( \pm 1 Day)}

- Collect study diary and remove completed pages (for storage in study files)

- Subjects will return used and unused investigational product, for reconciliation

- Review subject dosing compliance

- Dispense investigational product

- Return/dispense study diary

- Assessment of adverse events and concomitant medications/therapies

- Pain Assessment using Brief Pain Index 
- Administration of investigational product under supervision of site personnel

- Weight (kg) measurement

- A targeted physical examination may be completed if necessary (at the discretion of the Investigator), as required by a subject's signs and symptoms.

- At 1 hour ( $\pm 15 \mathrm{~min})$ after investigational product administration:

- 12-lead ECG

- Vital signs (pulse, resting blood pressure [must be resting for 10 minutes], respiration rate, and temperature)

- Blood samples for PK/PD analysis

- Blood samples for safety laboratory measures (hematology, chemistry and cardiac biomarkers)

\subsection{Day 8 Visit ( \pm 2 Days)}

- Collect study diary and remove completed pages (for storage in study files)

- Subjects will return used and unused investigational product, for reconciliation

- Review subject dosing compliance

- Dispense investigational product

- Return/dispense study diary

- Assessment of adverse events and concomitant medications/therapies

- Pain Assessment using Brief Pain Index

- Administration of investigational product under supervision of site personnel

- Weight (kg) measurement

- A targeted physical examination may be completed if necessary (at the discretion of the Investigator), as required by a subject's signs and symptoms.

- At 1 hour ( $\pm 15 \mathrm{~min}$ ) after investigational product administration:

- 12-lead ECG

- Vital signs (pulse, resting blood pressure [must be resting for 10 minutes], respiration rate, and temperature)

- Blood samples for safety laboratory measures (hematology, chemistry and cardiac biomarkers)

- Blood samples for PK/PD analysis 


\subsection{Day 14 (- 4 Days) Visit}

- Collect study diary and remove completed pages (for storage in study files)

- Assessment of adverse events and concomitant medications/therapies

- Review subject dosing compliance

- Administration of investigational product under supervision of site personnel

- Subjects will return used and unused investigational product, for reconciliation

- Return/dispense study diary

- Physical examination (including weight (kg) measurement)

- Bio-impedance Analysis

- Transthoracic Doppler Echocardiography (before CPX)

- $\mathrm{CPX}$

- Quality of Life and Pain Assessments

- Kansas City Cardiomyopathy Questionnaire

- Duke Activity Status Index

- Brief Pain Index

- At 1 hour ( $\pm 15 \mathrm{~min}$ ) after investigational product administration:

- Vital signs (pulse, resting blood pressure [must be resting for 10 minutes], respiration rate, and temperature)

- Blood sample for PK/PD analysis

- Blood samples for safety laboratory measures (hematology, chemistry and cardiac biomarkers)

\subsection{Day 28 ( \pm 2 Days) Follow-up Visit or Early Termination Visit}

- Collect study diary

- Assessment of adverse events and concomitant medications/therapies

- Physical examination (including weight ( $\mathrm{kg}$ ) measurement)

- Vital signs (pulse, resting blood pressure [must be resting for 10 minutes], respiration rate, and temperature)

- Blood samples for safety laboratory measures (hematology, chemistry and cardiac biomarkers) and PK/PD analysis 
- Urine pregnancy test for all females of childbearing potential

- Bio-impedance Analysis

- Transthoracic Doppler Echocardiography (before CPX)

- $\mathrm{CPX}$

- Quality of Life and Pain Assessments

- Kansas City Cardiomyopathy Questionnaire

- Duke Activity Status Index

- Brief Pain Index

- Early Termination (prior to Day 14) only: Return used and unused investigational product, for reconciliation

\subsection{Day 42 ( \pm 3 Days) Follow-up Telephone Call}

A safety follow-up call will be placed by site staff to each subject to assess if he/she has experienced any adverse events. Any adverse events that have occurred since the Day 28 visit will be recorded in study documentation and CRFs.

\subsection{Additional Follow-up for Adverse Events}

If a subject has an ongoing adverse event at the Day 28 visit, early termination visit or at the Day 42 follow-up call, he/she will be followed by the site personnel until resolution, stabilization or until the subject is lost to follow-up.

\subsection{Dosing Compliance Follow-up}

It is suggested (not required) that subjects be contacted by telephone for dosing compliance confirmation approximately every 1-2 days during the treatment period.

Note: If any study visit lasts over 4 hours, a light snack will be provided. 


\section{APPENDIX 2 AMERICAN HEART ASSOCIATION GUIDELINES FOR COLLECTION OF BLOOD PRESSURE}

- Subject should be seated comfortably, with back supported, legs uncrossed, and upper arm bared.

- Subject's arm should be supported at heart level.

- Cuff bladder should encircle 80 percent or more of the subject's arm circumference.

- Mercury column should be deflated at 2 to $3 \mathrm{~mm}$ per second.

- The first and last audible sounds should be recorded as systolic and diastolic pressure, respectively. Measurements should be given to the nearest $2 \mathrm{mmHg}$.

- Neither the subject nor the person taking the measurement should talk during the procedure.

Reference: Pickering TG, Hall JE, Appel LJ, Falkner BE, Graves J, Hill MN, et al.; Subcommittee of Professional and Public Education of the American Heart Association Council on High Blood Pressure Research. Recommendations for blood pressure measurement in humans and experimental animals. Part 1: blood pressure measurement in humans. Hypertension 2005;45:142-61. 


\section{APPENDIX $3 \quad$ CONTACT LIST}

\section{SPONSOR:}

Olatec Therapeutics LLC

800 Fifth Avenue, F1 25

New York, NY 10065

Office: +1 833-652-8321

Fax: +1 888-418-9873

Email: operations@olatec.com

\section{PRIMARY SPONSOR CONTACT:}

Cynthia Barrow

Olatec Therapeutics LLC

800 Fifth Avenue, F1 25

New York, NY 10065

Main Tel: +1 833-652-8321 x106

Mobile Tel: +1 919-414-2420

Fax: +1 888-418-9873

Email: cynthia.barrow@olatec.com and operations@olatec.com

\section{MEDICAL MONITOR:}

Curtis Scribner, MD

4287 Howe Street

Oakland, CA 94611

Main Tel: +1 833-652-8321 x108

Mobile Tel: +1 510-914-8368

Fax: +1 510-985-1665

E-mail: curt@clscribs.com and curt.scribner@olatec.com 